\title{
Neutralizing nanobodies bind SARS-CoV-2 spike RBD and block interaction with ACE2
}

\author{
Jiandong Huo, 1,2, Audrey Le Bas ${ }^{2,3}$, Reinis R. Ruza², Helen M. E. Duyvesteyn², Halina Mikolajek,

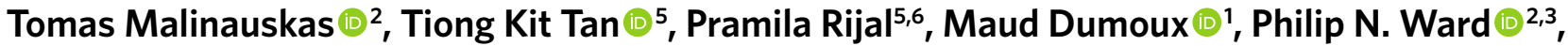 \\ Jingshan Ren², Daming Zhou' ${ }^{2}$, Peter J. Harrison ${ }^{2,3}$, Miriam Weckener', Daniel K. Clare ${ }^{4}$, \\ Vinod K. Vogirala4, Julika Radecke4 ${ }^{4}$ Lucile Moynié1, Yuguang Zhao ${ }^{1}{ }^{2}$, Javier Gilbert-Jaramillo ${ }^{7}$, \\ Michael L. Knight ${ }^{7}{ }^{7}$, Julia A. Tree ${ }^{8}$, Karen R. Buttigieg ${ }^{8}$, Naomi Coombes ${ }^{8}$, Michael J. Elmore ${ }^{8}$, \\ Miles W. Carroll ${ }^{8}$, Loic Carrique $\mathbb{( D}^{2}$, Pranav N. M. Shah $\mathbb{1}^{2}$, William James $\mathbb{}^{7}$, Alain R. Townsend ${ }^{5,6}$, \\ David I. Stuart $\mathbb{B}^{2,4}$, Raymond J. Owens ${ }^{1,2,3 凶}$ and James H. Naismith $\mathbb{1}^{1,2,3 凶}$
}

The SARS-CoV-2 virus is more transmissible than previous coronaviruses and causes a more serious illness than influenza. The SARS-CoV-2 receptor binding domain (RBD) of the spike protein binds to the human angiotensin-converting enzyme 2 (ACE2) receptor as a prelude to viral entry into the cell. Using a naive llama single-domain antibody library and PCR-based maturation, we have produced two closely related nanobodies, H11-D4 and H11-H4, that bind RBD ( $K_{\mathrm{D}}$ of 39 and $12 \mathrm{nM}$, respectively) and block its interaction with ACE2. Single-particle cryo-EM revealed that both nanobodies bind to all three RBDs in the spike trimer. Crystal structures of each nanobody-RBD complex revealed how both nanobodies recognize the same epitope, which partly overlaps with the ACE2 binding surface, explaining the blocking of the RBD-ACE2 interaction. Nanobody-Fc fusions showed neutralizing activity against SARS-CoV-2 (4-6 nM for H11-H4, $18 \mathrm{nM}$ for H11-D4) and additive neutralization with the SARS-CoV-1/2 antibody CR3022.

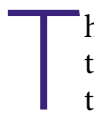
he SARS-CoV-2 virus is thought, based on sequence identity, to have crossed from bats to humans in $2019^{1}$. Similar to SARS-CoV-1 (2002-2003) and MERS-CoV (2012), SARS-CoV-2 presents as a respiratory disease but can progress into internal organs and cause organ failure ${ }^{2,3}$. A recent report from France estimates a fatality rate of $0.7 \%$ and a hospitalization rate of $3.6 \%{ }^{4}$. Both these rates are much higher in elderly populations ${ }^{4,5}$. Around $33 \%$ of those admitted to UK hospitals with COVID-19 have died ${ }^{6}$. Because SARS-CoV-2 also spreads rapidly in the naive human population 7 , the current COVID-19 pandemic has presented an unprecedented challenge to modern human society. Although there is currently no 'cure' or vaccine for the disease, passive immune therapy by transfusing critically ill COVID-19 patients with serum from COVID-19 convalescent individuals has been shown to improve clinical outcomes ${ }^{8,9}$. This would suggest that neutralization of the virus, even at a relatively late stage in the disease, may be a useful COVID-19 therapy.

The single-positive-strand RNA genome of SARS-CoV-2, like SARS-CoV, encodes four major structural proteins: spike, envelope, membrane and nucleocapsid. The spike protein comprises an $\mathrm{N}$-terminal (S1) subunit, which contains the roughly 200-residue receptor binding domain $(\mathrm{RBD})^{10,11}$, and a C-terminal subunit (S2), which contains the fusion protein ${ }^{12}$ (Fig. 1a). The RBD of
SARS-CoV-2 binds more tightly to the extracellular domain of angiotensin-converting enzyme 2 (ACE2) (Fig. 1a) than the homologous SARS-CoV-1 RBD ${ }^{13}$. The higher affinity results from sequence changes in RBD (Fig. 1b) and this has been proposed to underlie the higher transmissibility of SARS-CoV-2 ${ }^{14}$. Antibodies raised to the spike protein of SARS-CoV-1 can neutralize the virus both in vitro and in vivo, by binding to the RBD and blocking binding to $\mathrm{ACE} 2^{15}$. Unfortunately, most of these antibodies do not cross-react with the SARS-CoV-2 RBD ${ }^{13}$. The CR3022 antibody derived from a convalescent SARS-CoV-1 patient is cross-reactive to both SARS-CoV-1 and SARS-CoV-2 RBD (reported apparent $K_{\mathrm{D}}$ of $6 \mathrm{nM}$, ref. ${ }^{16}$ ). Two studies have reported crystal structures of CR3022 bound to SARS-CoV-2 RBD and show that the target epitope is distant from the ACE2 binding region ${ }^{17,18}$, which is consistent with the observation that CR3022 does not block RBD binding to ACE2. Another study on CR3022 has reported highly effective SARS-CoV-2 neutralizing activity that appears to arise from destabilization of the spike trimer, a novel mechanism for neutralizing SARS-CoV- $2^{18}$. Destabilization of viral proteins by antibodies has been observed for influenza ${ }^{19}$ and human immunodeficiency virus ${ }^{20}$.

Mammalian, including human, antibodies generally have two chains (heavy and light), but camelids, in addition to two-chain antibodies, also possess a single-heavy-chain antibody variant ${ }^{21}$.

\footnotetext{
'Structural Biology, The Rosalind Franklin Institute, Harwell Science \& Innovation Campus, Didcot, UK. '2Division of Structural Biology, University of Oxford,

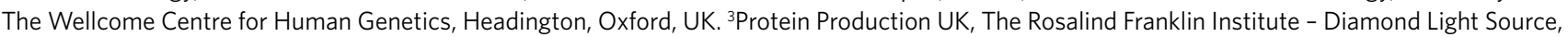
The Research Complex at Harwell, Harwell Science \& Innovation Campus, Didcot, UK. ${ }^{4}$ Diamond Light Source Ltd, Harwell Science \& Innovation Campus, Didcot, UK. ${ }^{5}$ MRC Human Immunology Unit, Weatherall Institute of Molecular Medicine, University of Oxford, John Radcliffe Hospital, Oxford, UK. ${ }^{6}$ Centre for Translational Immunology, Chinse Academy of Medical Sciences Oxford Institute, University of Oxford, Oxford, UK. ${ }^{7}$ Sir William Dunn School of Pathology, University of Oxford, Oxford, UK. ${ }^{8}$ National Infection Service, Public Health England, Porton Down, Salisbury, UK.

凶e-mail: raymond.owens@rc-harwell.ac.uk; naismith@strubi.ox.ac.uk
} 


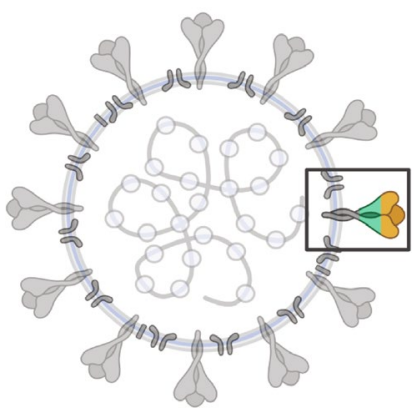

ACE2

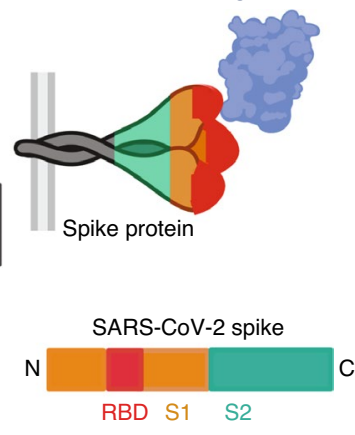

b

SARS-CoV-2 444 KGGYNYLYRLFRKSNLKPFERDIS四EIYQAGSTPCNGEGFNCYFES 494 SARS-CoV-1 431 TST GN KY YL HGKIRPEERDISNVPFSPDGK CT-PPALN YW N 480

C

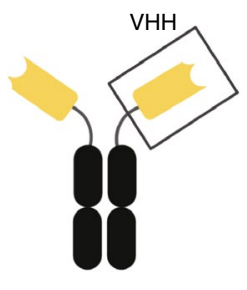

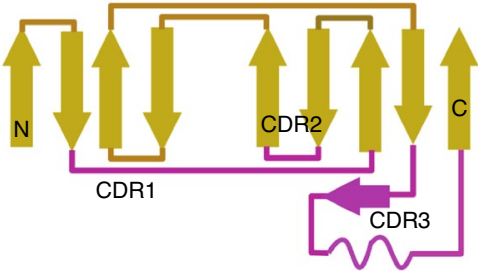

Fig. 1 | The spike protein of SARS-CoV-2 drives infection. a, Schematic of the spike protein of SARS-CoV-2. The spike protein is composed of S1 and S2 subunits. S1 contains the RBD (highlighted in red). Using the RBD, the trimeric spike molecule binds to ACE2 on human cells (a single ACE2 is shown in blue). b, RBD residues that are shared between SARS-CoV-2 and SARS-CoV-1 are highlighted in cyan (SARS-CoV-2, top) or in light gray text (SARS-CoV-1, bottom). Residues that contact ACE2 are highlighted in red for each sequence. Other sequence differences are in black text, with conservative substitutions indicated by colons (:) underneath. Residues in contact with the $\mathrm{H} 11-\mathrm{H} 4$ nanobody are boxed. c, Camelids have antibodies that are dimers of a single chain. The constant region is in black and the variable region in yellow. When the VHH domain is expressed on its own, it is termed a nanobody. A topology diagram shows that the nanobody is composed of two $\beta$-sheets. Three loops-complementarity-determining region 1 (CDR1), CDR2 and CDR3-control antigen binding and are highlighted in purple.

The antigen-specific variable portion of this single-chain antibody is termed the $\mathrm{VHH}$ domain and is commonly referred to as a nanobody (Fig. 1c). In addition to compatibility with phage display, nanobodies are small, stable and straightforward to produce ${ }^{21}$. As a result, they serve as alternates to conventional antibodies as diagnostics, imaging agents and structural biology tools ${ }^{21}$. The direct application of nanobodies in oncology and inflammatory diseases is being evaluated ${ }^{21,22}$, with caplacizumab ${ }^{23}$ approved for use. Nanobodies have been developed against SARS-CoV-1 and are currently being developed against SARS-CoV-2 $2^{24,25}$, both as research tools and potential therapeutics.

Here, we report the identification and characterization of two high-affinity nanobodies (H11-D4 and H11-H4) to the spike protein of SARS-CoV-2 that block the attachment of spike to ACE2 in vitro. Structural characterization of both nanobodies in complex with both full-length spike or with the RBD from SARS-CoV-2 has revealed that both target an epitope immediately adjacent to and slightly overlapping with the ACE2 binding region. Both nanobodies, when fused to immunoglobulin-G (IgG) Fc, neutralized live virus, with $\mathrm{H} 11-\mathrm{H} 4-\mathrm{Fc}$ showing a particularly high potency (50\% neutralization dose $\left(\mathrm{ND}_{50}\right)$ of $\left.4-6 \mathrm{nM}\right)$. H11-H4 also showed additive neutralization with CR3022 ${ }^{16}$. The nanobodies may have application on their own or in additive combinations with other antibodies in the treatment of severely ill COVID-19 patients.

\section{Results}

Identification of a spike-binding nanobody. We used purified RBD of the SARS-CoV-2 spike to identify its binding partners in a naive llama $\mathrm{VHH}$ library by in vitro phage display technology. We identified several nanobodies that bound to the RBD. The tightest binding nanobody, which we denoted $\mathrm{H} 11$, had a $K_{\mathrm{D}}$ of $<1 \mu \mathrm{M}$ (Extended Data Fig. 1a,b). Using a random mutagenesis approach, we identified two affinity matured mutants, H11-D4 and H11-H4, which differ from $\mathrm{H} 11$ and each other at five residues within CDR3 (Figs. $1 \mathrm{c}$ and 2a). H11-H4 and H11-D4 were shown to bind RBD by surface plasmon resonance (SPR), with an estimated $K_{\mathrm{D}}$ of $5 \mathrm{nM}$ and $10 \mathrm{nM}$, respectively (Fig. $2 \mathrm{~b}$ and Extended Data Fig. 1c,d). We performed an SPR-based competition assay in which ACE2-Fc was immobilized and then binding of RBD was monitored in the presence or absence of H11-H4 or H11-D4. In a similar experiment, we also monitored spike binding (instead of RBD). Both nanobodies inhibited the binding of both RBD and spike to ACE2 (Fig. 2c and Extended Data Fig. 1e). This suggested the nanobody epitope overlaps with the ACE2 binding site on the RBD of spike. When CR3022-Fc was immobilized and the binding of RBD measured, it was found that the RBD binds to CR3022, whether H11-H4 or H11-D4 was present or not (Fig. 2d and Extended Data Fig. 1f). This indicated that CR3022 and the nanobodies recognized non-overlapping epitopes on RBD. Repeating this experiment with spike also showed binding in the presence and absence of H11-H4 (Fig. 2d and Extended Data Fig. 1f).

The stoichiometry and thermodynamics of binding were characterized by isothermal titration calorimetry (ITC). H11-H4 binds to RBD with a $K_{\mathrm{D}}$ of $12 \pm 1.5 \mathrm{nM}$, while H11-D4 binds with a $K_{\mathrm{D}}$ of $39 \pm 2 \mathrm{nM}$; both showed a 1:1 stoichiometry (Fig. 2e and Extended Data Fig. 2a). When full-length trimeric spike was used, a single binding event was observed with a $1: 1$ nanobody:monomer $(3: 1$ nanobody:spike) stoichiometry and a $K_{\mathrm{D}}$ of $44 \pm 3 \mathrm{nM}$ for H11-H4 and $79 \pm 2 \mathrm{nM}$ for H11-D4 (Fig. $2 \mathrm{f}$ and Extended Data Fig. 2b). Despite increased enthalpy, H11-D4 bound more weakly than H11-H4 as a result of an increased entropic penalty upon binding. The same enthalpy entropy compensation is observed when comparing spike to RBD for both nanobodies. The spike protein has been proposed to exist in multiple conformational states in solution $^{26}$, yet ITC showed a simple binding curve (Fig. 2f). Either the nanobodies have bound equally well to all conformational states present or the equilibration between these states was faster than the binding event. The latter possibility seems less likely given the very high on rates (Fig. 2b).

A bivalent Fc-nanobody fusion competes with ACE2 for RBD binding. The nanobodies were fused to the Fc domain of human IgG1 to produce a homodimeric chimeric protein capable of bivalent binding (Fig. 3a). The ability of these constructs to block ACE2 binding to RBD was tested in two assays.

In the first assay, MDCK-SIAT1 cells stably expressing human ACE2 (MDCK-ACE2) were seeded on plates and the ability of various analytes (H11-H4-Fc, H11-D4-Fc, ACE2-Fc, CR3022 ${ }^{18}$ and VHH72-Fc) to block binding of RBD was measured (Fig. 3b). $\mathrm{VHH}_{72} 2^{25}$ is a nanobody isolated from a llama immunized with spike from SARS-CoV-1, which is cross-reactive against spike from SARS-CoV-2. The MDCK- ACE2 cell binding assay yielded a half-maximum inhibitory concentration $\left(\mathrm{IC}_{50}\right)$ of $61 \mathrm{nM}$ for H11-H4-Fc, $161 \mathrm{nM}$ for H11-D4-Fc and $262 \mathrm{nM}$ for VHH72-Fc ${ }^{25}$. 


$\begin{array}{llllll}\text { H11 } & 97 & \text { A } & \text { QTRVTRS } & \text { LLSDYATWPY } & 116 \\ \text { H11-H4 } & 97 & \text { A } & \text { QTHYVSY } & \text { LLSDYATWPY } & 116 \\ \text { H11-D4 } & 97 & \text { A } & \text { RTENVRS } & \text { LLSDYATWPY } & 116\end{array}$
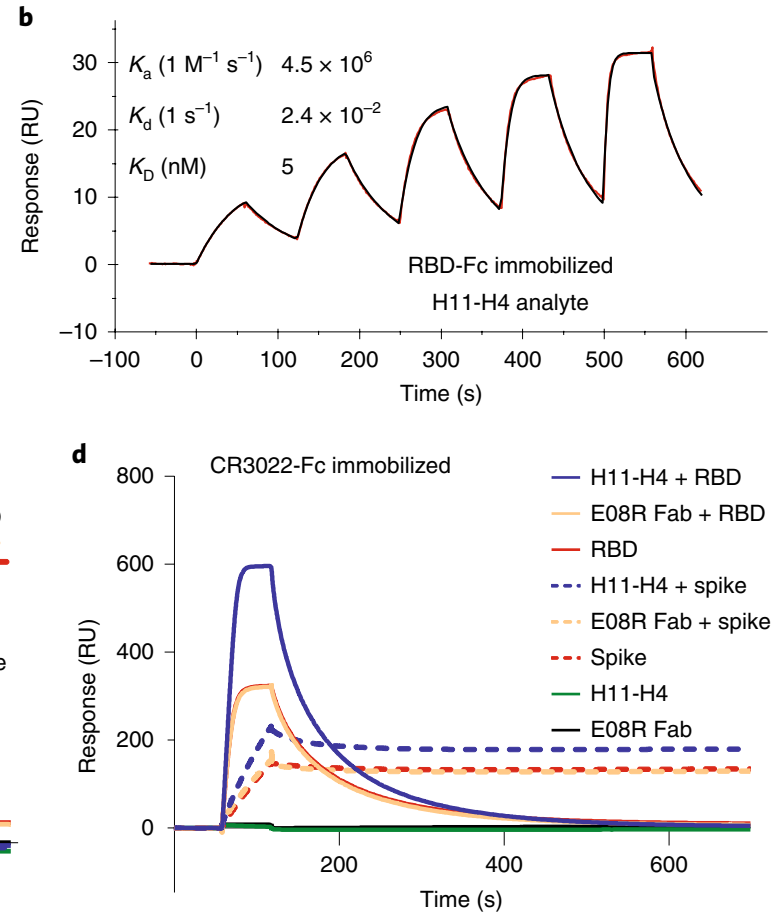

f

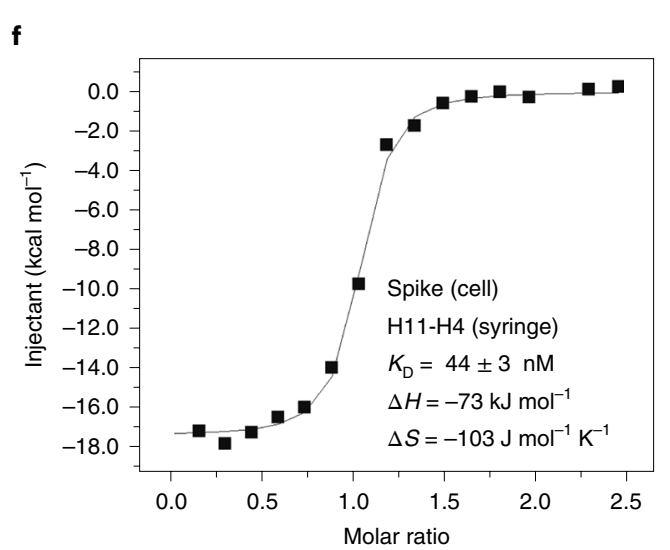

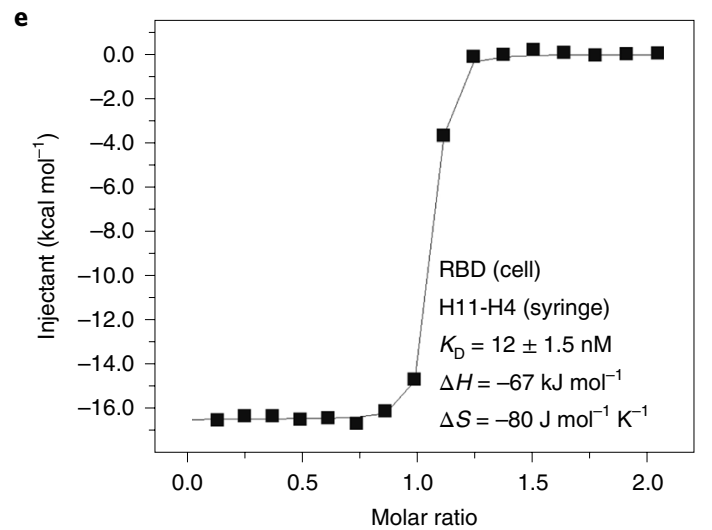

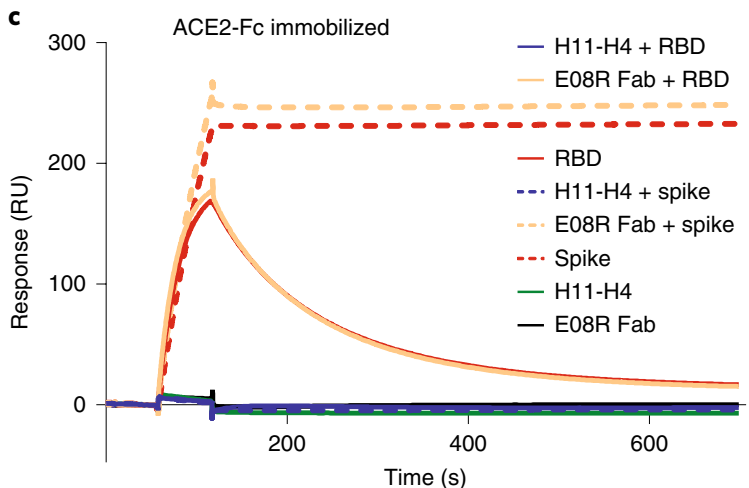

Fig. 2 | Laboratory-matured nanobodies bind to RBD and spike proteins with high affinity. a, Maturation by mutagenesis of CDR3 region of H11 resulted in $\mathrm{H} 11-\mathrm{D} 4$ and $\mathrm{H} 11-\mathrm{H} 4$. The five changes from the parent are shown in bold. b, SPR sensorgram showing that H11-H4 bound to RBD (immobilized as RBD-Fc on the chip) with $5 \mathrm{nM}$ affinity. A repeat experiment is shown in Extended Data Fig. 1c and H11-D4 data are provided in Extended Data Fig. 1d. c, RBD was bound by ACE2 (immobilized as ACE2-Fc on the chip). When RBD was pre-mixed with $\mathrm{H} 11-\mathrm{H} 4$, there was no binding, indicating that H11-H4 and ACE2 compete for binding to RBD. Similar results were observed using spike protein instead of RBD. The antibody E08R (anti-Caspr2 Fab) was used as a negative control. Data for H11-D4 are provided in Extended Data Fig. 1e. d, RBD was bound by CR3022 (immobilized as CR3022-Fc on the chip). When $\mathrm{RBD}$ was pre-mixed with $\mathrm{H} 11-\mathrm{H} 4$, binding occurred with similar on and off rates, indicating that $\mathrm{H} 11-\mathrm{H} 4$ and $\mathrm{CR} 3022$ recognize different epitopes on RBD. The response for the RBD H11-H4 mixture was larger, consistent with an H11-H4-RBD complex binding to CR3022. Antibody E08R was again used as a negative control. The spike protein shows binding to CR3022 in the presence or absence of H11-H4. Data for H11-D4 are provided in Extended Data Fig. 1f. e, ITC measurements show a $K_{D}$ of $12 \pm 1.5 \mathrm{nM}$ and a 1:1 ratio for H11-H4 and RBD association. Replicates and data for H11-D4 are provided in Extended Data Fig. 2a. f, ITC measurements show a $K_{\mathrm{D}}$ of $44 \pm 3 \mathrm{nM}$ and a 1:1 ratio for association between spike protein and H11-H4. Replicates and data for H11-D4 are provided in Extended Data Fig. $2 b$.

In the second competition assay, analytes (H11-H4-Fc, H11-D4-Fc, ACE2-Fc, CR3022 ${ }^{18}$, VHH72-Fc ${ }^{25}$ ) were assessed for their ability to block ACE2 binding to MDCK cells that expressed RBD on their surface (Fig. 3c). This assay yielded an $\mathrm{IC}_{50}$ of $34 \mathrm{nM}$ for H11-H4-Fc, $28 \mathrm{nM}$ H11-D4-Fc and $33 \mathrm{nM}$ for VHH72-Fc ${ }^{25}$. As expected, CR3022 does not show a strong response in either assay because it does not block the RBD-ACE2 interaction ${ }^{17,18}$.

H11-H4-Fc and H11-D4-Fc neutralize virus. The chimeric fusions were tested in a plaque reduction neutralization test at the Public
Health England Laboratory for SARS-CoV-2 virus, and showed an $\mathrm{ND}_{50}$ of $6 \mathrm{nM}$ for H11-H4-Fc (95\% CI 3-9nM) and $\mathrm{ND}_{50}$ of $18 \mathrm{nM}$ for H11-D4-Fc (95\% CI 9-68 nM) (Fig. 3d and Extended Data Fig. 3). H11-H4-Fc neutralization was replicated at Oxford University and yielded an $\mathrm{ND}_{50}$ of $4 \mathrm{nM}$. CR3022 was used as a positive control, and under these conditions an $\mathrm{ND}_{50}$ of $93 \mathrm{nM}$ was observed, similar to a previous report ${ }^{18}$ (Fig. 3e). The raw plates are shown in Extended Data Fig. 4 and we observed a small plaque phenotype in the presence of H11-H4-Fc but not in the positive control CR3022. A small plaque phenotype was noted in the PHE plates (Extended Data Fig. 3). 
a

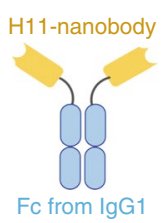

b

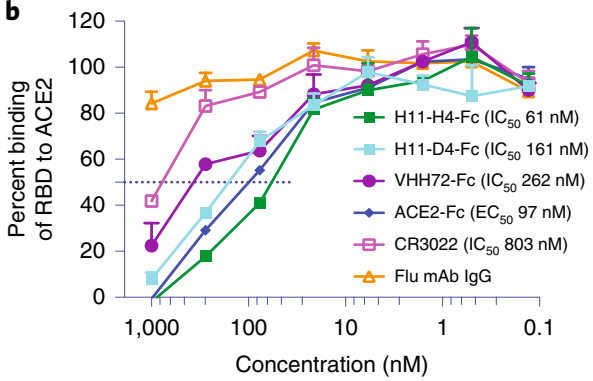

d

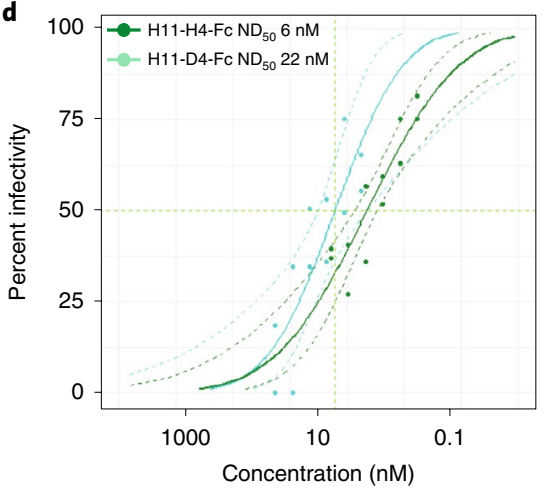

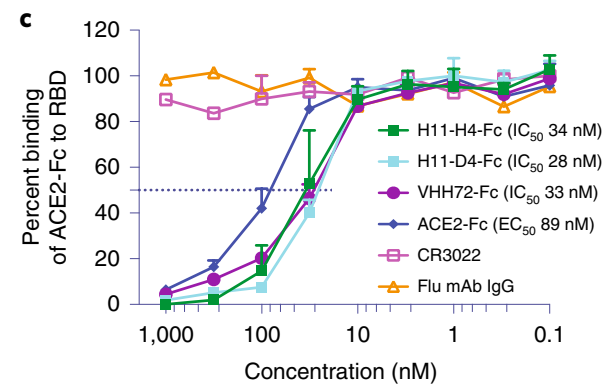

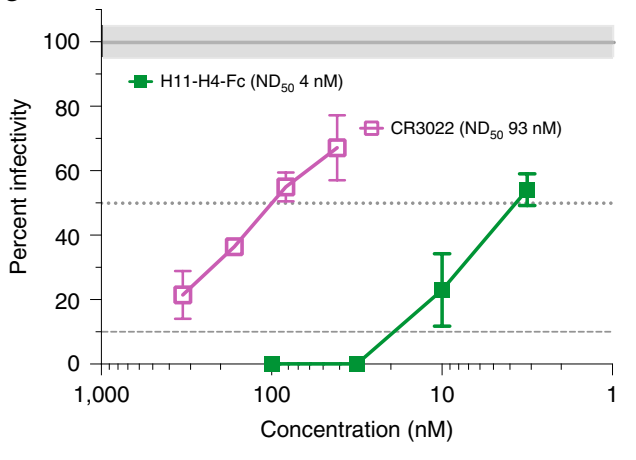

Fig. $\mathbf{3}$ | In vitro biological activity of H11-H4. a, Schematic of H11-H4-Fc, the lgG1 Fc (blue) fusion used in in vitro assays. b, Biotinylated RBD was mixed with analytes at various ratios and then added to MDCK-SIAT1 cells stably expressing human ACE2. The amount of biotinylated RBD bound was measured. Experiments were performed in duplicates with mean \pm s.d. shown. $\mathbf{c}$, Biotinylated ACE2-Fc was mixed with analytes at various ratios and then added to MDCK-SIAT1 cells stably expressing RBD. The amount of biotinylated ACE2-Fc bound was measured. Experiments were performed in duplicate with the mean \pm s.d. shown. d, $\mathrm{H} 11-\mathrm{H} 4-\mathrm{Fc}\left(\mathrm{ND}_{50}=6 \mathrm{nM}, 95 \%\right.$ confidence interval $\left.(\mathrm{Cl}) 3-9 \mathrm{nM}\right)$ and $\mathrm{H} 11-\mathrm{D} 4-\mathrm{Fc}\left(\mathrm{ND}_{50}=18 \mathrm{nM}, 95 \% \mathrm{Cl} 9-68 \mathrm{nM}\right)$ show potent neutralization of live wild-type virus. The $95 \% \mathrm{Cls}$ are shown as dashed lines. Detailed data plots are provided in Extended Data Fig. $3 a, b$ and an experimental plate in Extended Data Fig. 3c. e, $\mathrm{H} 11-\mathrm{H} 4-\mathrm{Fc}$ shows similar neutralization $\left(\mathrm{ND}_{50}=4 \mathrm{nM}\right)$ of live wild-type virus in a Vero cell-based assay in Oxford. CR3022 is shown as a positive control for this assay system and is similar to a previous report ${ }^{18}$. Data are presented as mean and s.d. of $n=2$ technical replicates. Experimental plates are shown in Extended Data Fig. 4a-c.

It should be noted that our assay method did not remove virus and neutralizing agent after incubation with cells, in line with UK standards. Some laboratories have reported a neutralization assay protocol where virus and neutralizing agent are removed during the assay, and precise protocol differences may be responsible for the reported difference in CR3022 neutralization ${ }^{17,18}$.

Structures of nanobody-spike and nanobody-RBD complexes. The nanobodies were each incubated at room temperature with a purified prefusion-stabilized ectodomain of the SARS-CoV-2 spike protein ${ }^{13}$ (spike(trimer):nanobody $=1: 4$ ) and then vitrified on cryo-EM grids. The cryo-EM single-particle structure of this variant of spike has been shown to be trimeric with a predominantly 'up-down-down' arrangement of the three RBDs ${ }^{13}$. After data collection and processing (Table 1 and Extended Data Figs. 5 and 6), the maps clearly identified additional density at all three RBDs in the H11-D4 and H11-H4 complexes (Extended Data Figs. 5 and 6). Improvement in the Coulomb potential maps allowed fitting of the nanobody into the additional density at each RBD in both structures (Table 1, Methods, Fig. 4a and Extended Data Figs. 5, 6 and 7a). The density for the nanobody bound to the 'up' RBD is weak, but still clearly discernible, while the density for the nanobodies bound to the 'down' RBDs is clearer (Extended Data Figs. 5 and 6).
The structures of H11-H4-spike (Fig. 4a) and H11-D4-spike complexes (Extended Data Fig. 7a) are indistinguishable given their resolution (Table 1). We focus our description here on the complex with the higher-affinity nanobody, H11-H4.

The region of the RBD in contact with the nanobody is ordered in the nanobody complex but is disordered in the EM prefusion stabilized holo spike structures (PDB 6VSB, 6VYB and 6VXX) ${ }^{13,26}$, precluding detailed analysis. However, we noted that in the nanobody-spike complex, the 'up' RBD (subunit A) makes contacts with the nanobody that is bound to 'down' RBD (subunit C) (Extended Data Fig. 7b) - contacts that are absent in the holo spike. These contacts have resulted in shifts of the RBD domains when compared to the non-complexed form ${ }^{13,26}$ (Extended Data Fig. 7c). Matching previous reports ${ }^{26}$, we have seen a mixture of two forms ('three down' and 'one up two down') on the grids for holo spike protein. In the presence of the nanobody, only the 'one up two down' form was observed, indicating that nanobody binding reduced conformational heterogeneity. We suggest the additional interactions are responsible for this observation and for the higher enthalpy and greater entropic penalty observed for nanobody binding to spike when compared to RBD (Fig. 2e,f).

Nanobodies rely on three variable loops, denoted CDR1, CDR2 and CDR3, to form the antigen-binding site (Fig. 1c). To gain 


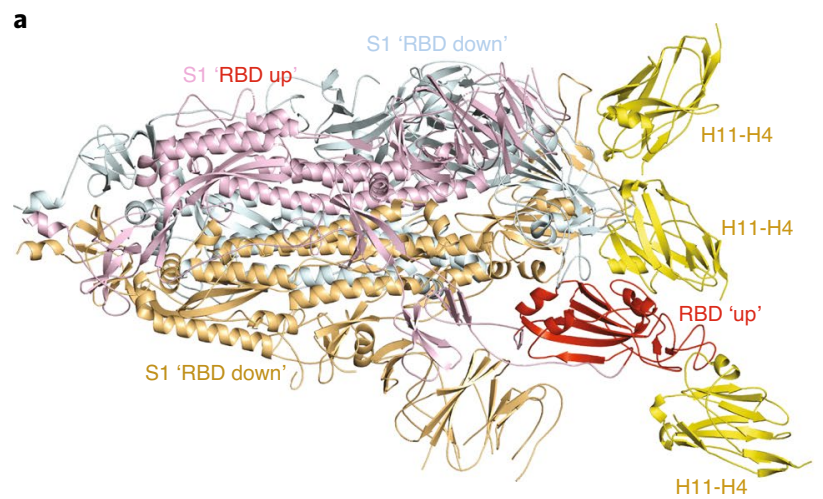

b

c
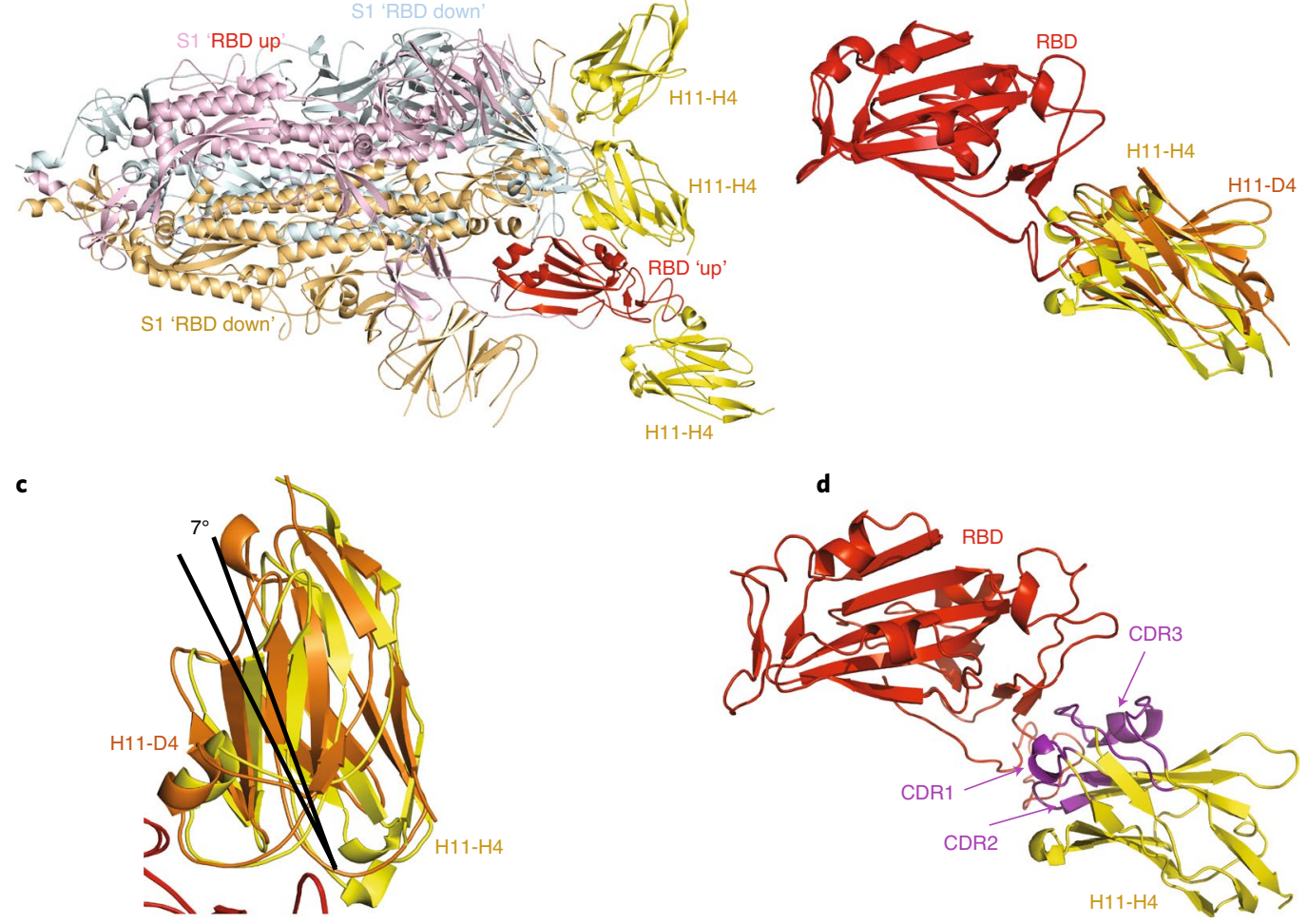

e

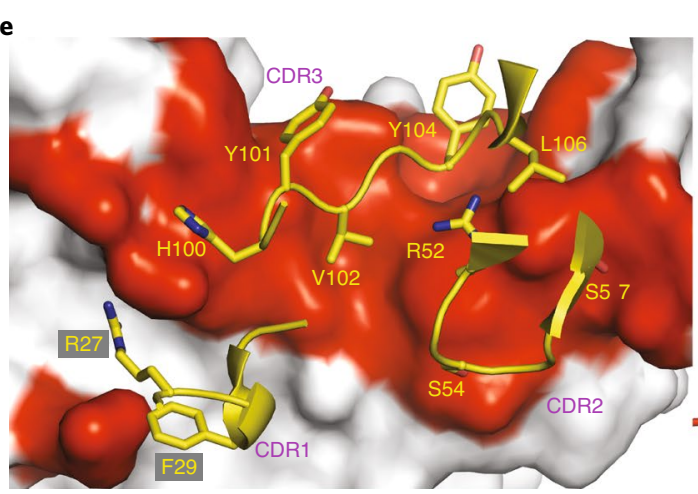

f

$\mathbf{g}$

h
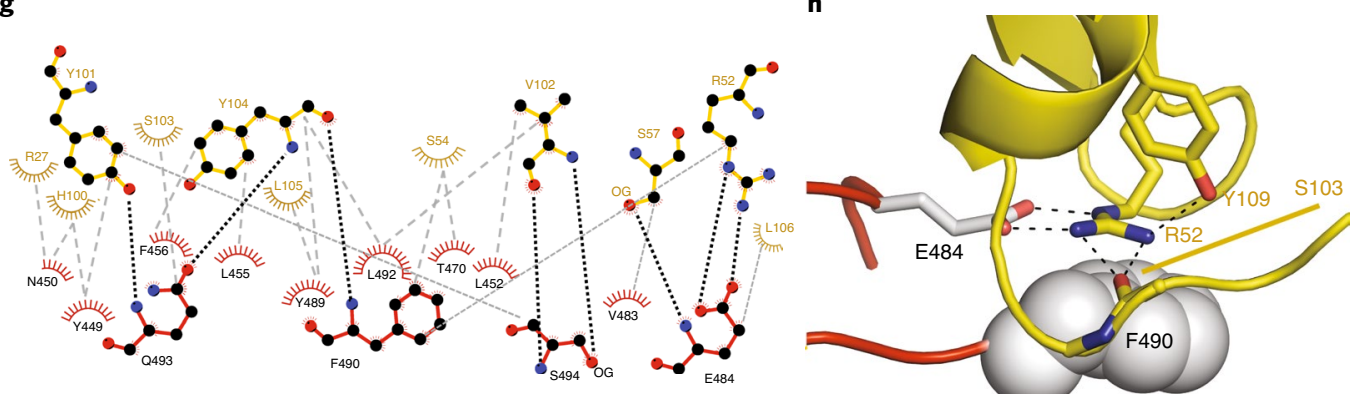

Fig. 4 | Structural biology of H11-H4 bound to spike and RBD. a, EM structure of spike (S1) trimer with each of three chains bound to one H11-H4 nanobody. The 'up' configured monomer is colored light pink, with the RBD highlighted in red. The other monomers are colored pale cyan and wheat throughout. Each spike monomer has bound one $\mathrm{H} 11-\mathrm{H} 4$ nanobody; the three $\mathrm{H} 11-\mathrm{H} 4$ nanobodies are colored yellow. b, Crystal structures of the $\mathrm{H} 11-\mathrm{H} 4-\mathrm{RBD}$ and H11-D4-RBD complexes were superimposed via the RBD (red), showing that both nanobodies recognize the same RBD epitope. H11-D4 is colored orange and $\mathrm{H} 11-\mathrm{H} 4$ yellow. $\mathbf{c}$, The superimposed nanobodies in $\mathbf{b}$ show a $7^{\circ}$ pivot between $\mathrm{H} 11-\mathrm{H} 4$ and H11-D4. d, Loops CDR1, CDR2 and CDR3 of H11-H4 control recognition and are highlighted in magenta. e, $\mathrm{A} 90^{\circ}$ rotation of the structure shown in d, showing the residues in contact with RBD. $\mathrm{H} 11-\mathrm{H} 4$ residues are labeled in yellow, with carbon atoms colored yellow, nitrogen atoms blue and oxygen atoms red. RBD is shown as a white surface with contact points ( $<4.0 \AA$ ) highlighted in red. $\mathbf{f}$, The same view as in $\mathbf{c}$, showing the RBD residues in contact with $\mathrm{H} 11-\mathrm{H} 4$ (omitted). RBD residues are labeled in black, with carbons colored in gray and other atoms as in c. $\mathbf{g}$, LigPlot ${ }^{42}$ of the interaction between H11-H4 (residues shown in gold, top) and RBD (residues shown in red, below). Hydrogen bonds are shown as black dashes and van der Waals interactions as light gray dashes. h, Arg52 of H11-H4 CDR2 stacks against the Phe490 of RBD and makes salt bridge contacts with Glu484. In addition, Arg52 makes hydrogen bonds with the main-chain Ser103 (side chain omitted) and Tyr109. H11-H4 residues are colored as in e. RBD residues are colored as in $\mathbf{f}$. 


\section{Table 1 | Cryo-EM data collection, refinement and validation statistics}

$\begin{array}{ll}\text { Spike-H11-D4 } & \text { Spike-H11-H4 } \\ \text { (EMD-11068, } & \text { (EMD-11218, } \\ \text { PDB 6Z43) } & \text { PDB 6ZHD) }\end{array}$

\section{Data collection and processing}

\begin{tabular}{|c|c|c|}
\hline Magnification & 105,000 & 81,000 \\
\hline Voltage (kV) & 300 & 300 \\
\hline Electron exposure $\left(\mathrm{e}^{-} / \AA^{2}\right)$ & 43 & 46 \\
\hline Defocus range $(\mu \mathrm{m})$ & $0.8-2.6$ & $1.0-3.0$ \\
\hline $\begin{array}{l}\text { Pixel size }(\AA / \text { pixel) } \\
\text { (super-resolution) }\end{array}$ & 0.415 & 0.53 \\
\hline Symmetry imposed & $C 1$ & $\mathrm{Cl}$ \\
\hline Initial particle images (no.) & 596,825 & 786,392 \\
\hline Final particle images (no.) & 305,513 & 126,938 \\
\hline Map resolution $(\AA)$ & 3.3 & 3.7 \\
\hline FSC threshold & 0.143 & 0.143 \\
\hline Map resolution range $(\AA)$ & $3.2-9.7$ & $3.7-7.0$ \\
\hline \multicolumn{3}{|l|}{ Refinement $^{a}$} \\
\hline Initial model used & PDB $6 V X X$ & PDB $6 Z 43$ \\
\hline Model resolution $(\AA)$ & 3.4 & 3.7 \\
\hline FSC threshold & 0.143 & 0.143 \\
\hline Model resolution range $(\AA)$ & $3.3-6.0$ & $3.7-6.0$ \\
\hline Map sharpening $B$ factor $\left(\AA^{2}\right)$ & -117 & -114 \\
\hline \multicolumn{3}{|l|}{ Model composition } \\
\hline Non-hydrogen atoms & 26,725 & 26,960 \\
\hline Protein residues & 3,351 & 3,419 \\
\hline \multicolumn{3}{|l|}{$B$ factors $\left(\AA^{2}\right)$} \\
\hline Protein & 119 & 183 \\
\hline \multicolumn{3}{|l|}{ R.m.s. deviations } \\
\hline Bond lengths $(\AA)$ & 0.005 & 0.006 \\
\hline Bond angles $\left({ }^{\circ}\right)$ & 0.58 & 1.28 \\
\hline \multicolumn{3}{|l|}{ Validation } \\
\hline MolProbity score & 1.43 & 1.53 \\
\hline Clashscore & 8.7 & 5.5 \\
\hline Poor rotamers (\%) & 0.1 & 0.9 \\
\hline \multicolumn{3}{|l|}{ Ramachandran plot } \\
\hline Favored (\%) & 94.8 & 96.4 \\
\hline Allowed (\%) & 5.1 & 3.6 \\
\hline Disallowed (\%) & 0.1 & 0.0 \\
\hline
\end{tabular}

aNanobody excluded from refinement.

insight into the molecular basis of recognition, crystal structures of the H11-H4-SARS-CoV-2 RBD complex and the H11-D4SARS-CoV-2 RBD complex were determined to resolutions of 1.85 and $1.80 \AA$, respectively (Table 2). Both crystal structures have a single copy of the complex in the asymmetric unit. Superposition of the two complexes has confirmed that both nanobodies recognize the same epitope (Fig. 4b). Comparison of the structures shows that the entire complex superimposes with a root-mean-square deviation (r.m.s.d.) of $1.0 \AA$ over $322 \mathrm{C} \alpha$ atoms, but the individual RBDs superimpose with an r.m.s.d. of $0.5 \AA$ over $195 \mathrm{C} \alpha$ atoms and the individual nanobodies with an r.m.s.d. of $0.4 \AA$ over $127 \mathrm{C} \alpha$ atoms. The higher r.m.s.d. for the complex arises from a $7^{\circ}$ pivot motion of the nanobodies with respect to each other (Fig. 4c). Given the very high degree of similarity between the complexes, we again focused the description on the H11-H4-RBD complex (Fig. 4d). There are differences compared to the H1-D4-RBD complex, due to sequence changes in the CDR3 loops, and a detailed description of the H1-D4-RBD interface is provided in Supplementary Figs. 1 and 2 and Supplementary Note 1 .

In the complex, the CDR1 loop of H11-H4 has contributed very little to the interface (Fig. 4e). From CDR2, residues Arg52, Ser54 and Ser57 have made contacts with RBD (Fig. 4d,e). From CDR3, His100 to Leu106, the region modified during maturation, made contacts with RBD (Fig. 4d,e). The surface on RBD that contacts H11-H4 is formed by Lys444 to Phe456 and Gly482 to Ser494 (Fig. 4f). These two stretches of RBD sequence comprise $90 \%$ of the buried surface area and make all the hydrogen bonds with H11-H4 (Fig. 4g). In addition to these direct contacts, there are multiple bridging water molecules. To our surprise, the PISA server ${ }^{27}$ does not identify either nanobody-RBD complex as stable.

Arg52 from the CDR2 of H11-H4 was found at the heart of a network of interactions, including RBD residues Glu484, with which it made a bivalent salt link, and Phe490, with which it made a $\pi$-cation interaction ${ }^{28}$ (Fig. 4h). Arg52 also forms hydrogen bonds to the backbone carbonyl of Ser103 and side chain of Tyr109 (Fig. 4h), which may stabilize the conformation of the CDR3 loop. The seven-residue stretch of the H11-H4 CDR3 region, which varied during maturation, contributes over $60 \%$ of the surface area buried by the complex and makes five hydrogen bonds to RBD (Fig. 4g).

Using the H11-D4-RBD complex, we created a model of three nanobodies bound to the 'down' (closed) form of the spike ${ }^{26}$ (Extended Data Fig. 7d). This model does not disclose any clashes, suggesting that the nanobody would bind to the spike protein in all its conformational states, consistent with the simple ITC curve (Fig. 2e).

The nanobody epitope compared to other RBD binders. Superposition of the RBD-ACE2 complex ${ }^{29,30}$ on the H11-H4-RBD complex reveals that $\mathrm{H} 11-\mathrm{H} 4$ would, consistent with biophysics (Fig. 2c), plate assays (Fig. 3b,c) and neutralization experiments (Fig. 3d), prevent ACE2 binding to RBD (Fig. 5a). This is due to van der Waals clashes, principally between regions of $\mathrm{H} 11-\mathrm{H} 4$ that are not in contact with the RBD and regions of ACE2 (also not in contact with RBD; Fig. 5a). Interestingly the contact surface of H11-H4 on RBD shows only a small overlap with the ACE2 contact surface (Figs. $1 \mathrm{~b}$ and $5 \mathrm{~b}$ ). Comparison with the RBD-ACE2 complex ${ }^{29,30}$ reveals that residues $445-500$ of RBD appear to move as a rigid unit upon H11-H4 binding (Extended Data Fig. 8a). The structure of the loop centered at Val483 of the RBD has changed upon binding of H11-H4 (Extended Data Fig. 8b).

Given the potential for additive and synergistic effects that can arise from combinations of antibodies and/or nanobodies that recognize different epitopes, crystals of ternary complexes H11-H4RBD-CR3022 (3.3 $\AA$ ) and H11-D4-RBD-CR3022 (2.7 $\mathrm{A})$ were obtained (Table 2). The structures are similar, and we focus on the higher-resolution H11-D4-RBD-CR3022 complex. As expected, the nanobody and the antibody bind to non-overlapping epitopes (Fig. 5c). Furthermore, comparison of H11-D4-RBD-CR3022 ternary complex with both the H11-D4-RBD complex and RBDCR3022 complex ${ }^{17,18}$ showed that binding of the nanobody does not perturb the recognition of the antibody and vice versa. This is consistent with biophysical analysis that shows CR3022 binds to $\mathrm{RBD}$ and to the nanobody-RBD complex equally well (Fig. $2 \mathrm{~d}$ and Extended Data Fig. 1f).

\section{Discussion}

It is assumed that, during the virus life cycle, the spike trimer exists in an equilibrium between the all 'down' configuration and mixed 'up down' states ${ }^{13}$. The spike protein can only bind to ACE2 with 
Table 2 | X-ray crystallography data collection and refinement statistics

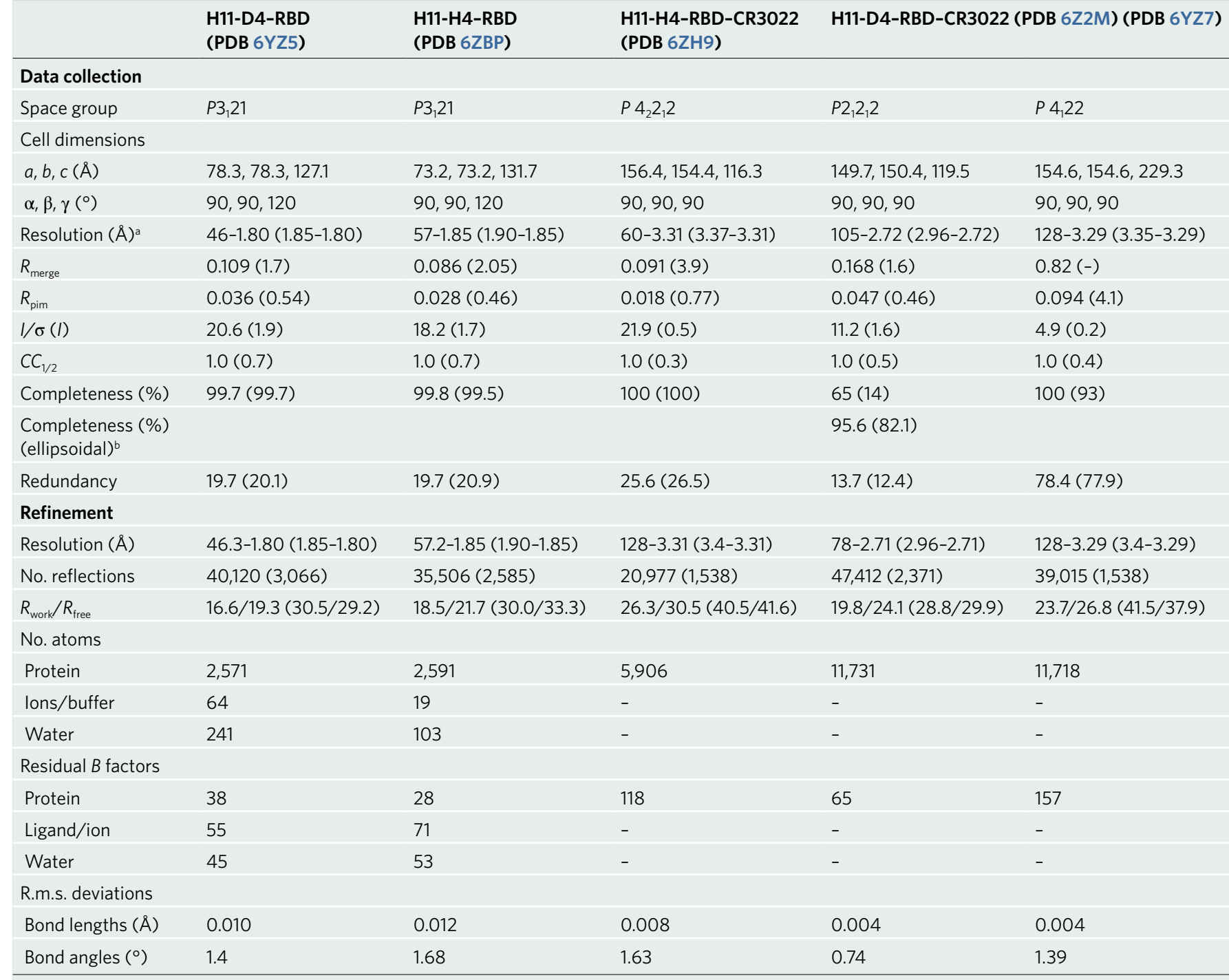

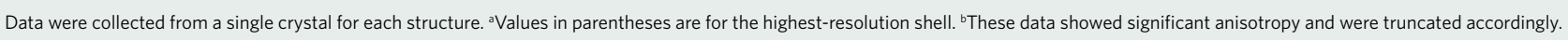

the RBD in the 'up' state ${ }^{11}$ and this results in dissociation of the trimer. SARS-CoV-2 spike binds to ACE2 with a 10 - to 20 -fold higher affinity $\left(K_{\mathrm{D}}\right.$ of $\left.\sim 15 \mathrm{nM}\right)$ than SARS-CoV-1 spike, a fact that has been proposed to drive its higher transmissibility ${ }^{13,31}$. Neutralizing antibodies that have been identified so far for SARS-CoV-1 bind to the RBD of the spike protein and many do so by blocking ACE2 binding $^{32}$, but CR3022 operates by a different mechanism ${ }^{18}$. We have identified two nanobodies, H11-H4 and H11-D4, which differ in sequence at five residues within the CDR3 loop (Fig. 2a) and have shown some subtle differences in properties (Figs. 2 and 3). Given that the H11-H4 nanobody has the higher affinity for RBD (Fig. 2e,f), the discussion focuses on this variant, but, unless explicitly stated, is equally valid for H11-D4.

We have shown that H11-H4 binds with high affinity to RBD (Fig. 2b,e,f), blocks ACE2 binding (Figs. $2 \mathrm{c}$ and $3 \mathrm{~b}, \mathrm{c}$ ) and neutralize the virus (Fig. 3d,e). Our analysis has suggested that H11-H4 would bind to both the 'all down' as well as 'two down one up' conformations of RBD within the spike (Fig. $4 \mathrm{a}$ and Extended Data Fig. 7c). The epitope on SARS-CoV-2 RBD that is recognized by H11-H4 overlaps only to a limited degree with the ACE2 binding region (Fig. 5a,b). This region of SARS-CoV-2 RBD has several sequence changes when compared to SARS-CoV-1 RBD (Fig. 1b). The Pro469-Pro470 turn in the SARS-CoV-1 RBD structure ${ }^{33}$ is very different to the structure at Val483-Glu484 in SARS-CoV-2. Additional sequence and structural changes between SARS-CoV-1 and SARS-CoV-2 (Tyr442 $\rightarrow$ Leu455, Trp476 $\rightarrow$ Phe490, Asn479 $\rightarrow$ Gln493) combine to present a very different epitope and would seem to preclude cross-reactivity of H11-H4. The lack of conservation of the H11-H4 epitope between SARS-CoV-1 and SARS-CoV-2 raises the possibility that SARS-CoV-2 variants may emerge that retain ACE2 receptor binding but are no longer recognized by $\mathrm{H} 11-\mathrm{H} 4$ or its relatives. At least some of the plausible escape mutations would perturb the position of Phe486, which inserts into a cleft in ACE2, an interaction important to the increased affinity of SARS-CoV-2 ${ }^{30}$. The rapid pipeline from naive library screen to maturation and thorough characterization does offer the possibility that new nanobodies could be generated against SARS-CoV-2 viruses that have escaped H11-H4.

The characterization of the cross-reactive (SARS-CoV-1, $K_{\mathrm{D}}=7 \mathrm{nM}$ and SARS-CoV-2, $K_{\mathrm{D}}=40 \mathrm{nM}$ ) nanobody VHH72 has been reported recently ${ }^{25}$. This nanobody blocks ACE2 binding and shows neutralization activity $\left(\mathrm{ND}_{50}=0.2 \mu \mathrm{g} \mathrm{ml}^{-1}\right)$ against the SARS-CoV-2 pseudovirus ${ }^{25}$. The crystal structure of the complex 


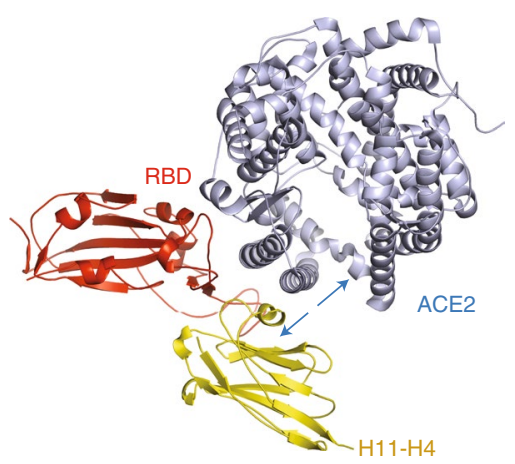

C

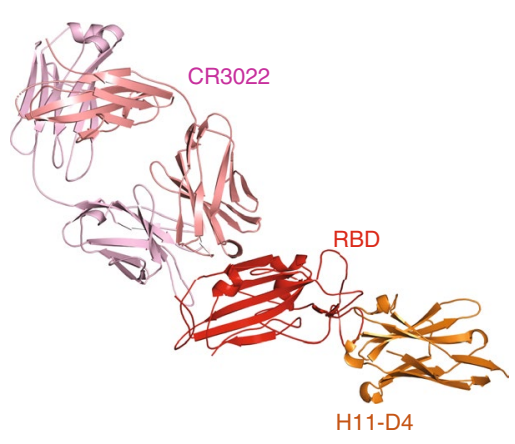

b

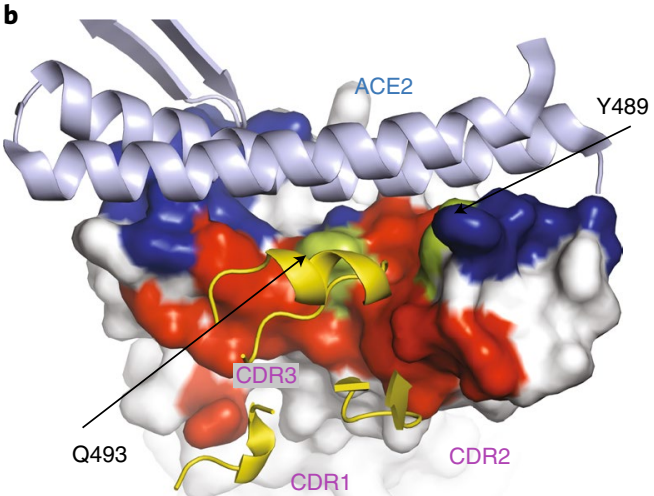

d

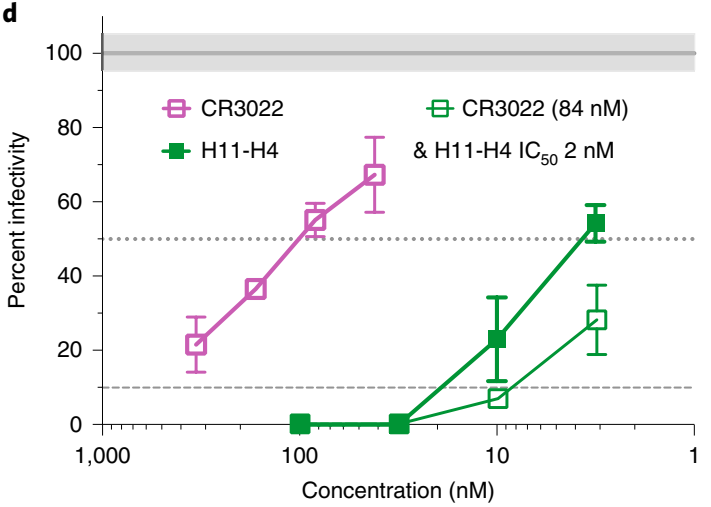

Fig. 5 | H11-H4 and CR3022 have different binding epitopes on RBD and show additive neutralization activities. a, Superposition using the RBD domains of the H11-H4-RBD complex (colored as in Fig. 4e) with the RBD-ACE2 complex (PDB 6MOJ29; ACE2 colored in pale blue). When H11-H4 is bound to $\mathrm{RBD}$, it would prevent ACE2 binding due to steric clashes. $\mathbf{b}$, The region of RBD that engages ACE2 only has a small overlap with the region recognized by $\mathrm{H} 11-\mathrm{H} 4$. The RBD is shown as a molecular surface, regions that only contact $\mathrm{ACE} 2$ are highlighted in dark blue, and those that only contact $\mathrm{H} 11-\mathrm{H} 4$ are in red. The two helices and turn of ACE2 that contact RBD are shown in cartoon representation and are colored in light blue. CDR1, CDR2 and CDR3 of $\mathrm{H} 11-\mathrm{H} 4$ are shown in cartoon form and are colored in yellow. Tyr489 and GIn493, which contribute considerably to both binding sites, are highlighted in light green. $\mathbf{c}$, The structure of the ternary complex H11-D4-RBD-CR3022 shows that the nanobody and antibody recognize entirely different epitopes. RBD is colored in red, CR3022 in pale pink and salmon, and H11-D4 in orange. d, Neutralization assay for H11-H4-Fc in the presence of CR3022 at a fixed concentration of $84 \mathrm{nM}$. The solid gray line represents the control values, with no neutralizing agent. Dashed lines are $50 \%$ and $10 \%$ of the control, respectively. Data are presented as mean and s.d. of $n=2$ technical replicates. The shift in the $\mathrm{H} 11-\mathrm{H} 4-\mathrm{Fc}$ neutralization curve and the measured ND 50 of $2 \mathrm{nM}$ indicate additivity. The experimental plates are shown in Extended Data Fig. 4d.

between VHH72 and RBD from SARS-CoV ${ }^{25}$ showed that VHH72 recognizes an epitope that is different from that bound by $\mathrm{H} 11-\mathrm{H} 4$ (Extended Data Fig. 9a). The epitope bound by VHH72 partly overlaps with the epitope bound by CR3022 ${ }^{18}$ (Extended Data Fig. 9b) and is found in a crystal contact between H11-H4 and RBD in the complex (Extended Data Fig. 9c,d). Another antibody, which, like CR3022, does not block ACE2 binding but neutralizes the virus, has also been published ${ }^{34}$, but there are no further structural details. Humanized nanobodies with potent neutralization activity against SARS-CoV-2 virus (most potent $\mathrm{ND}_{50}$ of $17-36 \mathrm{nM}$ in Vero cells) have been described ${ }^{35}$. Some, but not all, of these nanobodies blocked ACE2 binding and no molecular insights into their mode of action were reported ${ }^{35}$. A preprint has reported a llama antibody, Ty1, that neutralizes pseudovirus and blocks ACE2 binding ${ }^{36}$, but the coordinates of the EM structure are not available.

The use of convalescent serum has shown clinical promise in patients severely ill with SARS-CoV ${ }^{37}$ and most recently SARS-CoV- $2^{9}$; such passive immune therapy has a long history in medicine ${ }^{38}$. The use of laboratory-produced reagents avoids some of the infection risks that arise from use of human serum and can be administered in smaller volumes. The use of antibodies as therapies is well established but nanobodies have now entered clinical trials $^{21}$, with one, caplacizumab ${ }^{23}$, now licensed. The direct injection of a nanobody has also shown promise in a mouse model of cobra venom intoxication ${ }^{39}$. Camelid VHH domains are highly conserved with their human counterparts, and their immunogenicity has been proposed to be $l o w^{40}$, although humanization strategies are well developed $^{41}$.

To increase the in vivo half-life and enhance avidity, nanobodies can be multimerized by a variety of means ${ }^{22}$. For our in vitro binding assays (Fig. 3b,c) and neutralization experiments (Fig. 3d,e), we created a dimeric Fc fusion construct (Fig. 3a). Because the CR3022 antibody ${ }^{17,18}$ recognized a different epitope than H11-H4 (Figs. $2 \mathrm{~d}$ and $5 \mathrm{c}$ ), we investigated a combination of H11-H4 and CR3022 (CR3022 concentration fixed at $84 \mathrm{nM}$ ). Under these assay conditions, we observed evidence for an additive effect (Fig. 5d). Such additive combinations are a well-known strategy to reduce the propensity of the virus to escape by mutating.

This work establishes that nanobody maturation technology can be deployed to produce a highly neutralizing agent against an emerging viral threat in real time. The approach may be useful in identifying complementary epitopes to those identified by animal immunization approaches. The H11-H4 and H11-D4 nanobodies may find application in a cocktail of laboratory-synthesized neutralizing antibodies given for passive immunization of severely ill COVID-19 patients. 


\section{Online content}

Any methods, additional references, Nature Research reporting summaries, source data, extended data, supplementary information, acknowledgements, peer review information; details of author contributions and competing interests; and statements of data and code availability are available at https://doi.org/10.1038/ s41594-020-0469-6.

Received: 21 May 2020; Accepted: 26 June 2020; Published online: 13 July 2020

\section{References}

1. Menachery, V. D. et al. A SARS-like cluster of circulating bat coronaviruses shows potential for human emergence. Nat. Med. 21, 1508-1513 (2015).

2. Ronco, C., Reis, T. \& Husain-Syed, F. Management of acute kidney injury in patients with COVID-19. Lancet Resp. Med. 8, 738-742 (2020).

3. Jaiswal, N. K. \& Saxena, S. K. in Medical Virology: From Pathogenesis to Disease Control: Coronavirus Disease 2019 (COVID-19) 141-150 (Springer, 2020).

4. Salje, H. et al. Estimating the burden of SARS-CoV-2 in France. Science https://doi.org/10.1126/science.abc3517 (2020); erratum https://doi. org/10.1126/science.abd4246

5. Adams, M. L., Katz, D. L. \& Grandpre, J. Population-based estimates of chronic conditions affecting risk for complications from Coronavirus Disease, United States. Emerg. Infect. Dis. https://doi.org/10.3201/eid2608.200679 (2020).

6. Docherty, A. B. et al. Features of 20133 UK patients in hospital with covid-19 using the ISARIC WHO Clinical Characterisation Protocol: prospective observational cohort study. BMJ 369, m1985 (2020).

7. Kucharski, A. J. et al. Early dynamics of transmission and control of COVID-19: a mathematical modelling study. Lancet Infect. Dis. 20, 553-558 (2020).

8. Bloch, E. M. et al. Deployment of convalescent plasma for the prevention and treatment of COVID-19. J. Clin. Invest. 130, 2757-2765 (2020).

9. Shen, C. et al. Treatment of 5 critically ill patients with COVID-19 with convalescent plasma. JAMA 323, 1582-1589 (2020).

10. Wan, Y., Shang, J., Graham, R., Baric, R. S. \& Li, F. Receptor recognition by the novel coronavirus from Wuhan: an analysis based on decade-long structural studies of SARS coronavirus. J. Virol. 94, e00127-20 (2020).

11. Yan, R. et al. Structural basis for the recognition of SARS-CoV-2 by full-length human ACE2. Science 367, 1444-1448 (2020).

12. Li, F. Structure, function and evolution of coronavirus spike proteins. Annu. Rev. Virol. 3, 237-261 (2016).

13. Wrapp, D. et al. Cryo-EM structure of the 2019-nCoV spike in the prefusion conformation. Science 367, 1260-1263 (2020).

14. Hoffmann, M. et al. SARS-CoV-2 cell entry depends on ACE2 and TMPRSS2 and is blocked by a clinically proven protease inhibitor. Cell 181, 271-280 (2020).

15. Zhu, Z. et al. Potent cross-reactive neutralization of SARS coronavirus isolates by human monoclonal antibodies. Proc. Natl Acad. Sci. USA 104, 12123-12128 (2007)

16. Tian, X. et al. Potent binding of 2019 novel coronavirus spike protein by a SARS coronavirus-specific human monoclonal antibody. Emerg. Microbes Infect. 9, 382-385 (2020).

17. Yuan, M. et al. A highly conserved cryptic epitope in the receptor-binding domains of SARS-CoV-2 and SARS-CoV. Science 368, 630-633 (2020).

18. Huo, J. et al. Neutralization of SARS-CoV-2 by destruction of the prefusion spike. Cell Host Microbe https://doi.org/10.1016/j.chom.2020.06.010 (2020).

19. Winarski, K. L. et al. Antibody-dependent enhancement of influenza disease promoted by increase in hemagglutinin stem flexibility and virus fusion kinetics. Proc. Natl Acad. Sci. USA 116, 15194-15199 (2019).

20. Kim, A. S., Leaman, D. P. \& Zwick, M. B. Antibody to gp41 MPER alters functional properties of HIV-1 Env without complete neutralization. PLoS Pathog. 10, e1004271 (2014).
21. Jovčevska, I. \& Muyldermans, S. The therapeutic potential of nanobodies. BioDrugs 34, 11-26 (2020).

22. Chanier, T. \& Chames, P. Nanobody engineering: toward next generation immunotherapies and immunoimaging of cancer. Antibodies (Basel) 8, 13 (2019).

23. Peyvandi, F. et al. Caplacizumab reduces the frequency of major thromboembolic events, exacerbations and death in patients with acquired thrombotic thrombocytopenic purpura. J. Thromb. Haemost. 15, 1448-1452 (2017).

24. Zhou, P. et al. A pneumonia outbreak associated with a new coronavirus of probable bat origin. Nature 579, 270-273 (2020).

25. Wrapp, D. et al. Structural basis for potent neutralization of Betacoronaviruses by single-domain camelid antibodies. Cell 181, 1436-1441 (2020).

26. Walls, A. C. et al. Structure, function and antigenicity of the SARS-CoV-2 spike glycoprotein. Cell 181, 281-292 (2020).

27. Krissinel, E. \& Henrick, K. Detection of protein assemblies in crystals. CompLife 3695, 163-174 (2005).

28. Gallivan, J. P. \& Dougherty, D. A. Cation $-\pi$ interactions in structural biology. Proc. Natl Acad. Sci. USA 96, 9459-9464 (1999).

29. Lan, J. et al. Structure of the SARS-CoV-2 spike receptor-binding domain bound to the ACE2 receptor. Nature 581, 215-220 (2020).

30. Shang, J. et al. Structural basis of receptor recognition by SARS-CoV-2. Nature 581, 221-224 (2020).

31. Song, W., Gui, M., Wang, X. \& Xiang, Y. Cryo-EM structure of the SARS coronavirus spike glycoprotein in complex with its host cell receptor ACE2. PLoS Pathog. 14, el007236 (2018).

32. Suits, M. D., Sperandeo, P., Dehò, G., Polissi, A. \& Jia, Z. Novel structure of the conserved Gram-negative lipopolysaccharide transport protein A and mutagenesis analysis. J. Mol. Biol. 380, 476-488 (2008).

33. Li, F., Li, W., Farzan, M. \& Harrison, S. C. Structure of SARS coronavirus spike receptor-binding domain complexed with receptor. Science 309, 1864-1868 (2005).

34. Wang, C. et al. A human monoclonal 1 antibody blocking SARS-CoV-2 infection. Nat. Commun. 11, 2251 (2020).

35. Chi, X. et al. Humanized single domain antibodies neutralize SARS-CoV-2 by targeting spike receptor binding domain. Preprint at bioRxiv https://doi. org/10.1101/2020.04.14.042010 (2020).

36. Hanke, L. et al. An alpaca nanobody neutralizes SARS-CoV-2 by blocking receptor interaction. Preprint at bioRxiv https://doi. org/10.1101/2020.06.02.130161 (2020).

37. Mair-Jenkins, J. et al. The effectiveness of convalescent plasma and hyperimmune immunoglobulin for the treatment of severe acute respiratory infections of viral etiology: a systematic review and exploratory meta-analysis. J. Infect. Dis. 211, 80-90 (2015).

38. Slifka, M. K. \& Amanna, I. J. Passive immunization. Plotkin's Vaccines 8 , $84-95$ (2018).

39. Richard, G. et al. In vivo neutralization of $\alpha$-cobratoxin with high-affinity llama single-domain antibodies (VHHs) and a VHH-Fc antibody. PLOS ONE 8, e69495 (2013).

40. Klarenbeek, A. et al. Camelid Ig V genes reveal significant human homology not seen in therapeutic target genes, providing for a powerful therapeutic antibody platform. MAbs 7, 693-706 (2015).

41. Vincke, C. et al. General strategy to humanize a camelid single-domain antibody and identification of a universal humanized nanobody scaffold. J. Biol. Chem. 284, 3273-3284 (2009).

42. Laskowski, R. A. \& Swindells, M. B. LigPlot+: multiple ligand-protein interaction diagrams for drug discovery. J. Chem. Inf. Model 51, 2778-2786 (2011).

Publisher's note Springer Nature remains neutral with regard to jurisdictional claims in published maps and institutional affiliations.

() The Author(s), under exclusive licence to Springer Nature America, Inc. 2020, corrected publication 2021 


\section{Methods}

Protein production. Nanobody sequences (DNA and protein), protein constructs and production are described in full in Supplementary Note 2. All primers used in this work are listed in Supplementary Table 1. Plasmids encoding the nanobodies described here are available at Addgene (www.addgene.org).

Screening of a VHH library to identify molecules that bind to SARS-CoV-2 RBD . A VHH phage display library (Abcore) constructed in the vector pADL-20c and comprising $\sim 1 \times 10^{10}$ independent clones was inoculated into $2 \times T Y A$ (2XTY supplemented with $100 \mu \mathrm{g} \mathrm{ml}^{-1}$ ampicillin) and infected with M13 helper phage to obtain a library of VHH-presenting phages. Phages displaying VHHs specific for the SARS-CoV-2 RBD were enriched after two rounds of biopanning on $50 \mathrm{nM}$ and $5 \mathrm{nM}$ of RBD, respectively, by capturing with Dynabeads M-280 (Thermo Fisher Scientific). For each round of panning, the Dynabeads and phages were first blocked with StartingBlock (PBS) blocking buffer (Thermo Fisher Scientific) for $30 \mathrm{~min}$. The phages were incubated with the RBD for $1 \mathrm{~h}$ and then 5 min with the Dynabeads (Thermo Fisher Scientific), and subsequently washed six times with PBS supplemented with $0.05 \%$ Tween 20 and once with PBS. The retained phages were eluted through incubation with TBSC buffer (10 mM Tris pH 7.4, $137 \mathrm{mM} \mathrm{NaCl}^{1} \mathrm{mM} \mathrm{CaCl}_{2}$ ) and $1 \mathrm{mg} \mathrm{m}^{-1}$ trypsin (Sigma-Aldrich) for $30 \mathrm{~min}$. The collected phages were amplified in exponentially growing TG1 Escherichia coli cells and plated on 2xTY agar plates supplemented with $100 \mu \mathrm{g} \mathrm{ml}^{-1}$ ampicillin. Enrichment after each round of panning was determined by plating the cell culture with 10 -fold serial dilutions. After the second round of panning, 93 individual clones were picked to inoculate 2 xTYA and were grown overnight at $37^{\circ} \mathrm{C}$, while shaking at 250 r.p.m. The next day, the overnight culture was used to inoculate 2xTYA and infected with M13 helper phage to obtain clonal VHH-presenting phages.

Enzyme-linked immunosorbent assays to quantitate initial binding. The wells of microtiter plates (Greiner high and medium binding) were coated with $5 \mu \mathrm{gl}^{-1}$ neutravidin in PBS pH 7.4 overnight at $4{ }^{\circ} \mathrm{C}$. The next day, the wells were coated with $50 \mathrm{nM}$ biotinylated RBD, then blocked with $3 \%$ milk powder in PBS pH 7.4. Supernatant of clonal phage was added into each well, binding was detected by incubating the wells with horseradish peroxidase (HRP)-conjugated anti-M13 (GE Healthcare). After washing, $100 \mu \mathrm{l}$ of TMB substrate (SeraCare) was added and absorbance at $405 \mathrm{~nm}$ was measured with a microplate absorbance reader.

Affinity maturation of nanobody H11. Mutations in the CDR3 of nanobody H11 were introduced by PCR using seven pairs of forward and reverse primers as shown in Supplementary Table 1 (H11_AM_CDR3_F1-7 in combination with H11_AM_CDR3_R1-7). The mutated fragments were amplified with primers H11_Phd_F and H11_Phd_R, digested with SfiI restriction enzyme and cloned into pADL-23c phagemid (Antibody Design Laboratories). The ligated vector was transformed into TG1 cells by electroporation to give a phage library consisting of $\sim 2 \times 10^{9}$ independent clones. Two rounds of biopanning of the library were carried out on $5 \mathrm{nM}$ and $1 \mathrm{nM} \mathrm{RBD}$, respectively, as described above, and positive phage was identified by ELISA and sequencing.

\section{Surface plasmon resonance and isothermal titration calorimetry. SPR} experiments were performed using a Biacore T200 system (GE Healthcare). All assays were performed using a Sensor Chip Protein A (GE Healthcare), with a running buffer of $\mathrm{PBS}$ pH 7.4 supplemented with $0.005 \% \mathrm{vol} / \mathrm{vol}$ surfactant $\mathrm{P} 20$ (GE Healthcare) at $25^{\circ} \mathrm{C}$

To determine the binding affinity of nanobody $\mathrm{H} 11$ for the SARS-CoV-2 RBD, RBD-Fc was immobilized onto the sample flow cell of the sensor chip. The reference flow cell was left blank. Nanobody H11 was injected over the two flow cells at a range of eight concentrations prepared by serial twofold dilutions from $2.5 \mu \mathrm{M}$, at a flow rate of $30 \mu \mathrm{min}^{-1}$, with an association time of $60 \mathrm{~s}$ and a dissociation time of $60 \mathrm{~s}$. The data were fitted to a 1:1 binding model and to calculate $K_{\mathrm{D}}$ using GraphPad Prism 8.

To determine the binding kinetics between the SARS-CoV-2 RBD and nanobody H11-H4/H11-D4, RBD-Fc was immobilized onto the sample flow cell of the sensor chip. The reference flow cell was left blank. Nanobody H11-H4/H11-D4 was injected over the two flow cells at a range of five concentrations prepared by serial twofold dilutions from $50 \mathrm{nM}$, at a flow rate of $30 \mu \mathrm{min}^{-1}$ using a single-cycle kinetics program with an association time of $60 \mathrm{~s}$ and a dissociation time of $60 \mathrm{~s}$. Running buffer was also injected using the same program for background subtraction. All data were fitted to a 1:1 binding model using Biacore T200 Evaluation Software 3.1.

In the competition assay where $\mathrm{CR} 3022-\mathrm{Fc}$ or ACE2-Fc was used as the ligand, $\sim 1,000 \mathrm{RU}$ of CR3022-Fc or ACE2-Fc was immobilized. The following samples were injected: (1) a mixture of $1 \mu \mathrm{M}$ nanobody H11-H4/H11-D4 and 0.1 $\mu \mathrm{M}$ RBD; (2) a mixture of $1 \mu \mathrm{M}$ E08R (anti-Caspr2 Fab) Fab and $0.1 \mu \mathrm{M} \mathrm{RBD}$; (3) $0.1 \mu \mathrm{M}$ RBD; (4) a mixture of $1 \mu \mathrm{M}$ nanobody H11-H4/H11-D4 and $0.1 \mu \mathrm{M}$ spike; (5) a mixture of $1 \mu \mathrm{M}$ E08R Fab and $0.1 \mu \mathrm{M}$ spike; (6) $0.1 \mu \mathrm{M}$ spike; (7) $1 \mu \mathrm{M}$ nanobody H11-H4/H11-D4; (8) $1 \mu \mathrm{M}$ E08R Fab. All injections were performed with an association time of $60 \mathrm{~s}$ and a dissociation time of $600 \mathrm{~s}$. All curves were plotted using GraphPad Prism 8.
ITC measurements were carried out using an iTC200 MicroCalorimeter (GE Healthcare) at $25^{\circ} \mathrm{C}$. Spike, RBD and nanobody were prepared and dialyzed in the same buffer, that is, PBS. Nanobody was titrated into spike or RBD solution corresponding to $\sim 72 \mu \mathrm{M}$ nanobody and $6 \mu \mathrm{M}$ spike or $250 \mu \mathrm{M}$ nanobody and $25 \mu \mathrm{M}$ RBD. Each experiment consisted of an initial injection of $0.4 \mu$ followed by 16 injections of $2.4 \mu \mathrm{l}$ of nanobody solution into the cell containing either spike or RBD, while stirring at 750 r.p.m. Data acquisition and analysis were performed using the Origin scientific graphing and analysis software package (OriginLab). Data analysis was performed by generating a binding isotherm and best fit using the following parameters: $n$ (number of sites), $\Delta H$ (calories per mole), $\Delta S$ (calories per mole per degree) and $K$ (binding constant in $\mathrm{mol}^{-1}$ ). Following data analysis, $K$ was converted to the dissociation constant $\left(K_{\mathrm{D}}\right.$, in $\left.\mathrm{nM}\right)$.

\section{ACE2 blocking and neutralization experiments. MDCK-SIAT1 cells were} stably transfected with codon-optimized human ACE2 cDNA (NM_021804.1) using a second-generation lentiviral vector system and fluorescent activated cell sorting (FACS) for highly expressing population. Cells $\left(3 \times 10^{4}\right.$ per well $)$ were seeded the day before the assay on a flat-bottomed 96-well plate. RBD-6H (amino acids 340-538; NITN.GPKK) was chemically biotinylated using EZ-link sulfo-NHS-biotin (A39256, Life Technologies). A serial half-log dilution (ranging from $1 \mu \mathrm{M}$ to $0.1 \mathrm{nM}$ ) of analytes and controls was performed in a U-bottomed 96-well plate in a volume of $30 \mu \mathrm{l}$. An equal volume of $25 \mathrm{nM}$ biotinylated RBD was added and $50 \mu \mathrm{l}$ of each of the resulting mixtures were added to the MDCK-ACE2 cells for $1 \mathrm{~h}$. A second layer of streptavidin-HRP (S911, Life Technologies) diluted 1:1,600 in PBS/0.1\% BSA (37525, Thermo Fisher Scientific) was then added and incubated for $1 \mathrm{~h}$. Plates were then washed with PBS four times, and the signal was developed by adding POD substrate (11484281001, Roche) for $5 \mathrm{~min}$ before stopping with $1 \mathrm{M} \mathrm{H}_{2} \mathrm{SO}_{4}$. Plates were read at an optical density at $450 \mathrm{~nm}\left(\mathrm{OD}_{450}\right)$ on a Clariostar plate reader. The control analyte (a non-blocking anti influenza N1 antibody) was used to obtain the maximum signal and PBS-only wells were used to determine background. Graphs were plotted as percent binding of biotinylated $\mathrm{RBD}$ to ACE2. Binding $\%=(X-\min ) /(\max -\min ) \times 100$ where $X=$ measurement of the competing component, $\mathrm{min}=$ buffer without binder biotinylated RBD-6H, $\max =$ biotinylated RBD- $6 \mathrm{H}$ alone. The $\mathrm{IC}_{50}$ values of the nanobodies against ACE2 were determined using nonlinear regression [inhibitor] versus normalized response curve fit using GraphPad Prism 8 .

MDCK-SIAT1 cells were stably transfected with RBD (amino acids 340-538 NITN.GPKK) fused to the transmembrane and cytoplasmic domain of hemagglutinin H7 (A/HongKong/125/2017) (EPI977395) via a short linker for surface expression (sequence TGSGGSGKLSSGYKDVILWFSFGASCFILLAIV MGLVFICVKNGNMRCTICI*) using a second-generation lentiviral vector system. RBD-expressing cells were FACS sorted using the CR3022 antibody. Cells $\left(3 \times 10^{4}\right.$ per well) were seeded the day before the assay on a flat-bottomed $96-$ well plate. ACE2-Fc was biotinylated as above. A serial half-log dilution (ranging from $1 \mu \mathrm{M}$ to $0.1 \mathrm{nM}$ ) of analytes and controls was performed in a U-bottomed 96-well plate in a volume of $30 \mu \mathrm{l}$, then $30 \mu \mathrm{l}$ of biotinylated Ace2-Fc at $5 \mathrm{nM}$ was added to the titrated analytes. Cells were washed with PBS and $50 \mu \mathrm{l}$ of each mixture of ACE2 and an analyte was transferred to the cells and incubated for $1 \mathrm{~h}$ at room temperature. Cells were then washed with PBS and incubated for $1 \mathrm{~h}$ with the second-layer streptavidin-HRP (S911, Life Technologies), diluted to 1:1,600, and developed as above. Graphs were plotted as percent binding of biotinylated ACE2 to RBD. Binding $\%=(X-\min ) /(\max -\min ) \times 100$ where $X=$ measurement of the competing component, $\mathrm{min}=$ buffer without binder biotinylated ACE2-Fc, $\max =$ biotinylated $\mathrm{ACE} 2-\mathrm{Fc}$ alone. $\mathrm{IC}_{50}$ values of the nanobodies against ACE2 were determined using nonlinear regression [inhibitor] versus normalized response curve fit using GraphPad Prism 8. Non-biotinylated ACE2-Fc-6H and VHH72-Fc were used as positive controls.

Plaque reduction neutralization tests at Public Health England used SARS-CoV-2 (Australia/VIC01/2020) ${ }^{43}$, which was diluted to a concentration of 933 p.f.u. $\mathrm{ml}^{-1}(70$ p.f.u. $/ 75 \mu \mathrm{l})$ and mixed 50:50 in minimal essential medium (MEM; Life Technologies) containing 1\% FBS (Life Technologies) and $25 \mathrm{mM}$ HEPES buffer (Sigma) with doubling antibody dilutions in a 96-well V-bottomed plate. The plate was incubated at $37^{\circ} \mathrm{C}$ in a humidified box for $1 \mathrm{~h}$ to allow neutralization to take place. Afterwards, the virus-antibody mixture was transferred into the wells of a twice Dulbecco's PBS-washed 24-well plate containing confluent monolayers of Vero E6 cells (ECACC 85020206, PHE) that had been cultured in MEM containing 10\% (vol/vol) FBS. Virus was allowed to adsorb onto cells at $37^{\circ} \mathrm{C}$ for a further hour in a humidified box, then the cells were overlaid with MEM containing 1.5\% carboxymethyl cellulose (Sigma), $4 \%$ (vol/vol) FBS and $25 \mathrm{mM}$ HEPES buffer. After five days incubation at $37^{\circ} \mathrm{C}$ in a humidified box, the plates were fixed overnight with $20 \%$ formalin/PBS ( $\mathrm{vol} / \mathrm{vol}$ ), washed with tap water and then stained with $0.2 \%$ crystal violet solution (Sigma) and plaques were counted. A mid-point probit analysis (written in R programming language for statistical computing and graphics) was used to determine the dilution of antibody required to reduce SARS-CoV-2 viral plaques by $50 \%\left(\mathrm{ND}_{50}\right)$ compared with the virus-only control $(n=5)$. The script used in $\mathrm{R}$ was based on a previously reported source script ${ }^{44}$. Antibody dilutions were run in duplicate and an internal positive control for the PRNT assay was also run in duplicate using a sample of heat-inactivated $\left(56^{\circ} \mathrm{C}\right.$ for $30 \mathrm{~min}$ ) human MERS convalescent serum known to 
neutralize SARS-CoV-2 (National Institute for Biological Standards and Control, UK). The plates are shown in Extended Data Fig. 3c.

Plaque reduction neutralization tests in Oxford were performed using passage 4 of SARS-CoV-2 Victoria/01/2020 $0^{43}$ using the established methodology ${ }^{45}$. In brief, virus stock $\left(9.75 \times 10^{4}\right.$ p.f.u. $\left.\mathrm{ml}^{-1}\right)$ was diluted by 10 and by 100 in Dulbecco's modified Eagle's medium containing 1\% FBS (D1; $100 \mu \mathrm{l})$, mixed with nanobody-Fc $(100 \mu \mathrm{l})$ diluted in $\mathrm{D} 1$ so as give a final concentrations of $\mathrm{H} 11-\mathrm{H} 4$ at $100,32,10,3.2 \mathrm{nM}$ for measurement. As a positive control, solutions with CR3022 333, 167, 84 and 42 nM were prepared. Each experiment was performed in triplicate in a 24 -well tissue culture plate. The plate was incubated at room temperature for $30 \mathrm{~min}$ and $0.5 \mathrm{ml}$ of a single cell suspension of Vero E6 cells in $\mathrm{D} 1$ at $5 \times 10^{5} \mathrm{ml}^{-1}$ was added. The plates were incubated for a further $2 \mathrm{~h}$ at $37^{\circ} \mathrm{C}$ before being overlain with $0.5 \mathrm{ml}$ of $\mathrm{D} 1$ supplemented with carboxymethyl cellulose (1.5\%). The resulting cultures were incubated for a further four days at $37^{\circ} \mathrm{C}$ before plaques were revealed by staining the cell monolayers with Amido Black in acetic acid/methanol (Extended Data Fig. 4a-d). To probe whether CR3022 and H11-H4 were additive, solutions of $\mathrm{H} 11-\mathrm{H} 4$ at $100,32,10$ and $3.2 \mathrm{nM}$ were each incubated for 30 min with CR3022 at a final concentration of $84 \mathrm{nM}$. The resulting mixtures were analyzed as described above in triplicate experiments and the wells are shown in Extended Data Fig. 4d.

Cell lines. Oxford neutralization used Vero Ccl-81 (from a stock that was originally from ATCC). PHE neutralization used Vero E6 cells purchased from ECACC. Cell-based competition assays used MDCK-SIAT 1 cells derived from a commercial source (Sigma-Aldrich). All mammalian protein expressions were performed with purchased 293Expi cells (Thermo Fisher Scientific) and E. coli cells.

Nanobody complex with spike, preparation and cryo-electron microscopy data collection. Purified spike protein in $10 \mathrm{mM}$ HEPES, pH $8,150 \mathrm{mM} \mathrm{NaCl}$ was incubated with $\mathrm{H} 11-\mathrm{H} 4$ purified in $50 \mathrm{mM}$ Tris, $\mathrm{pH} 7,150 \mathrm{mM} \mathrm{NaCl}$, at a molar ratio of 1:3.6 (spike trimer:nanobody) at $16^{\circ} \mathrm{C}$ overnight. Spike protein was used at a final concentration of $1 \mathrm{mg} \mathrm{ml}^{-1}$. The mixture was centrifuged at $21,000 \mathrm{~g}$ at $16^{\circ} \mathrm{C}$ before grid preparation. For H11-D4-spike, a mixture in a molar ratio of 1:6 (spike trimer:nanobody) was incubated at $20^{\circ} \mathrm{C}$ for $10 \mathrm{~min}$.

A $3 \mu$ l volume of the resulting H11-D4-spike sample was then applied to a holey carbon-coated 200 mesh copper grid (C-Flat, CF-2/1, Protochips) that had been freshly glow-discharged on high for $20 \mathrm{~s}$ (Plasma Cleaner PDC-002-CE, Harrick Plasma). Excess liquid was removed by blotting for $6 \mathrm{~s}$ with a blotting force of -1 using Vitrobot filter paper (grade 595, Ted Pella) at $4.5^{\circ} \mathrm{C}$ and $100 \%$ relative humidity. Blotted grids were then immediately plunge-frozen using a Vitrobot Mark IV system(Thermo Fisher Scientific).

Frozen grids were first screened on a Glacios microscope operating at $200 \mathrm{kV}$ (Thermo Fisher Scientific) before imaging on a Titan Krios G2 (Thermo Fisher Scientific) at $300 \mathrm{kV}$. Videos ( 40 frames each) were collected in compressed tiff format on a $\mathrm{K} 3$ detector (Gatan) in super-resolution counting mode using a custom EPU version 2.5 (Thermo Fisher Scientific) (Table 1). Motion correction and alignment of $2 \times$ binned super-resolution movies was performed using Relion (v3.1 $)^{46}$ with a $5 \times 5$ patch-based alignment. Contrast transfer function (CTF) estimation of full-frame non-weighted micrographs was performed using GCTF (v1.06), and non-template-driven particle picking was then performed within cryoSPARC (v2.14.1-live) ${ }^{47}$ followed by multiple rounds of two-dimensional (2D) classification. The resulting $2 \mathrm{D}$ class averages consistent with spike trimer were used for template-driven particle picking before further rounds of $2 \mathrm{D}$ and $3 \mathrm{D}$ classification with $C 1$ symmetry. The resulting map from the most populous class was then sharpened in cryoSPARC before conversion to Relion-format star files using custom pyEM scripts ${ }^{48}$ (csparc2star.py, https://github.com/asarnow/pyem) for further CTF refinement within Relion.

An initial model for spike was generated using PDB $6 \mathrm{VXX}^{26}$ and rigid body fitted into the map using Chimera ${ }^{49}$ followed by Coot $^{50}$. The H11-D4-RBD crystal structure was superimposed onto the naked spike model in Coot and checked for fit in the density. S1/S2 domains split into subdomains for each subunit (residues 27-307; 308-321 and 591-700; 322-333 and 529-590; 701-1147) were then independently rigid body fitted in $\operatorname{Coot}^{50}$, before a final real-space refinement with PHENIX ${ }^{51}$, with hydrogen atoms added using ReadySet ${ }^{51}$, resulting in a final correlation coefficient of 0.8. The H11-D4-RBD crystal structure was used as reference structure restraints during refinement of the spike due to the density. Rounds of manual inspection in $\mathrm{Coot}^{50}$ and real-space refinement with PHENIX ${ }^{51}$ resulted in the final model. Data processing and refinement statistics are shown in Table 1.

For the H11-H4 spike sample, SPT Labtech prototype 300 mesh 1.2/2.0 nanowire grids with a highly reproducible rectangular bar cross-section were used. The grids were glow-discharged on low for $90 \mathrm{~s}$ (plasma cleaner PDC-002-CE, Harrick Plasma) to activate the nanowires. Approximately $6 \mathrm{nl}$ of the complex were applied to the grids using a Chameleon EP system (SPT Labtech) at $81 \%$ relative humidity and ambient temperature.

Frozen grids were screened and then data collected using a Titan Krios G2 system (Thermo Fisher Scientific) equipped with a Bioquatum-K3 detector (Gatan) operated at $300 \mathrm{kV}$. Videos (50 frames each) were collected in compressed tiff format in super-resolution counting mode using a custom EPU version 2.5 (Thermo Fisher Scientific).
Processing of videos up to 2D classification was done automatically using the Relion_IT.py processing pipeline implemented at eBIC. In detail, motion correction and alignment of $2 \times$ binned super-resolution movies were performed using Relion (v3.08) ${ }^{46}$ with a $5 \times 5$ patch-based alignment. CTF estimation of full-frame non-weighted micrographs was performed using GCTF (v1.06) and non-template-driven particle picking was then performed within crYOLO $^{52}$ followed by $2 \mathrm{D}$ classification. The best $2 \mathrm{D}$ classes clearly showing details consistent with the spike complex were selected for further processing. 3D classification was performed using emd_21374 low-pass-filtered to $60 \AA$. Initially the data were processed as $C 3$ but relaxed to $C 1$ as the RBD and nanobody densities were poor. The best $C 13 \mathrm{D}$ class was selected for further refinement, CTF refinement and particle polishing within Relion.

The coordinates from the spike-H11-D4 structure were rigid-body-docked into the spike-H11-H4 cryo-EM density in Chimera $^{49}$ and then refined with multiple rounds of jelly body refinement using RefMac5 via CCP-EM GUI ${ }^{53,54}$ and manual intervention with Coot resulted in a final correlation coefficient of 0.78 . Due to the limited resolution of the nanobody density in the cryo-EM map, the refined nanobody structure was replaced by the docked H11-H4-RBD crystal structure in the final model. Finally, the nanobodies were docked as rigid bodies into the cryo-EM density using Chimera ${ }^{49}$ to optimize their position. Data processing and refinement statistics are shown in Table 1.

H11-D4-RBD-CR3022 and H11-H4-RBD-CR3022 crystallography. Purified RBD, Fab CR3022 and H11-D4 were mixed together at a molar ratio of 1:1:1 to a final concentration of $\sim 7 \mathrm{mg} \mathrm{ml}^{-1}$ and incubated at room temperature for $1 \mathrm{~h}$. Initial screening was performed in 96-well plates using the nanoliter sitting-drop vapor diffusion method. The best crystals were grown in conditions containing $0.1 \mathrm{M}$ sodium citrate tribasic dihydrate, $\mathrm{pH} 5.0$ and $10 \%$ (wt/vol) polyethylene glycol (PEG) 6000

Purified RBD, Fab CR3022 and nanobody H11-H4 were mixed together at a molar ratio of 1:1:1.1, incubated at room temperature for $1 \mathrm{~h}$ and run on a gel filtration column. Initial screening was performed in 96-well plates using the nanoliter sitting-drop vapor diffusion method. The best crystals were grown by mixing $0.1 \mu \mathrm{l}$ of the $20 \mathrm{mg} \mathrm{ml}^{-1} \mathrm{H} 11-\mathrm{H} 4-\mathrm{RBD}-\mathrm{CR} 3022$ complex with $0.1 \mu \mathrm{l}$ of the crystallization buffer as above. Crystals were soaked in cryoprotectant containing $70-75 \%$ reservoir solution and $20-25 \%$ glycerol for a few seconds, then mounted in loops and frozen in liquid nitrogen before data collection at beamline I03 of Diamond Light Source, UK.

Two crystal forms for H11-D4-RBD-CR3022 were obtained (Table 2). For the first form collected, three crystals, $360^{\circ}$ each, were merged to give a final dataset to 3.3-Å resolution with 78-fold redundancy. A second form appeared later and yielded $2.7 \AA$ from a single crystal, although the data were anisotropic. A single crystal of H11-H4-RBD-CR3022 was collected.

Data were indexed, integrated and scaled with the automated data-processing program Xia2-dials ${ }^{55,56}$. The crystal structure of the first crystal of the H1-D4RBD-CR3022 complex was solved by molecular replacement using the known RBD-CR3022 structure (PDB 6YLA ${ }^{18}$ ) and the known structure of the nanobody 9G8 (PDB 4KRP ${ }^{57}$ ). The high-resolution structures of the H11-D4-RBD and H11-H4-RBD complexes then became available and were used in subsequent solutions. The electron density H11-H4-RBD-CR3022 was, as seen in the low-resolution H11-D4-RBD-CR3022 structure, poor for the nanobody-a reflection of the relatively low resolution of the study.

Model rebuilding was done with Coot $^{50}$, initially refined with PHENIX ${ }^{51}$ then with REFMAC $5^{58}$ aided by PDB-REDO ${ }^{59}$, MOLPROBITY $^{60}$ and the TLSMD server $^{61}$

H11-H4-RBD and H11-D4-RBD crystallography. Each nanobody was mixed with $8.7 \mathrm{mg}$ of RBD at $2.9 \mathrm{mg} \mathrm{ml}^{-1}$ at a molar ratio of nanobody:RBD of 1.1:1 and the complex was incubated for $3 \mathrm{~h}$ in a cold room under agitation at 2 r.p.m. RBD in the complex was deglycosylated by the addition of $0.4 \mathrm{mg}$ of EndoH glycosidase and incubated overnight at room temperature, under agitation at 2 r.p.m. The mixture was then concentrated to $1 \mathrm{ml}$ with a $5-\mathrm{kDa}$ molecular weight cutoff (MWCO) concentrator and injected for gel filtration using a Superdex 200 10/300 system (GE) in $50 \mathrm{mM}$ Tris $\mathrm{pH} 7,150 \mathrm{mM} \mathrm{NaCl}$. The peak fractions were pooled and concentrated using a 5-kDa MWCO concentrator to $10 \mathrm{mg} \mathrm{ml}^{-1}, 18 \mathrm{mg} \mathrm{ml}^{-1}$ and $29 \mathrm{mg} \mathrm{ml}^{-1}$.

Crystallization screening was performed on the Diamond/RCaH/RFI HTP crystallization facility at Harwell. Crystals of H11-D4-RBD were grown at $20^{\circ} \mathrm{C}$ using the sitting-drop vapor diffusion method by mixing $0.2 \mu \mathrm{l}$ of the $18 \mathrm{mg} \mathrm{ml}^{-1}$ complex with $0.1 \mu \mathrm{l}$ of the crystallization buffer containing $0.2 \mathrm{M}$ sodium acetate trihydrate, 0.1 M MES pH 6.0, 20\% wt/vol PEG 8000. H11-D4-RBD crystals grew overnight and were flash-cooled in a solution containing the mother liquor with $30 \%$ (vol/vol) ethylene glycol. Crystals of $\mathrm{H} 11-\mathrm{H} 4-\mathrm{RBD}$ were grown at $20^{\circ} \mathrm{C}$ using the sitting-drop vapor diffusion method by mixing $18 \mathrm{mg} \mathrm{ml}^{-1}$ of complex with $0.1 \mu \mathrm{l}$ of the crystallization buffer containing $0.2 \mathrm{M}$ lithium sulfate, $0.1 \mathrm{M}$ Bis Tris pH 5.5 and 25\% wt/vol PEG 3350. H11-H4-RBD crystals grew overnight and were flash-cooled in a solution containing the mother liquor with $30 \%(\mathrm{vol} / \mathrm{vol})$ PEG 400. Diffraction data were also collected and processed at beamline I03 at Diamond Light Source. The H11-D-RBD structure was solved by molecular 
replacement ${ }^{62}$ using the RBD and H11-D4 monomers from the ternary complex above. Refinement was carried out as described above for the ternary complex. The H11-H4-RBD complex was solved using the H11-D4-RBD complex. Statistics for $\mathrm{X}$-ray data collection and structure refinement are provided in Table 2. Electron densities for both complexes are shown in Extended Data Fig. 8c,d.

Reporting Summary. Further information on research design is available in the Nature Research Reporting Summary linked to this Article.

\section{Data availability}

The coordinates and structure factors were deposited in the wwPDB with accession nos. PDB 6ZH9 (H11-H4-RBD-CR3022), 6ZBP (H11-H4-RBD), 6YZ7 and 6Z2M (H11-D4-RBD-CR3022) and 6YZ5 (H11-D4-RBD). EM maps and models are deposited in the EMDB and wwPDB under accession codes EMD-11218 and PDB 6ZHD (prefusion spike with H11-H4); EMD-11068 and PDB $6 Z 43$ (prefusion spike with H11-D4). The underlying biophysical data and raw images for neutralization are provided in the Extended Data. Nanobody sequences are provided in Supplementary Note 2.

\section{References}

43. Caly, L. et al. Isolation and rapid sharing of the 2019 novel coronavirus (SARS-CoV-2) from the first patient diagnosed with COVID-19 in Australia. Med. J. Aust. 212, 459-462 (2020).

44. Johnson, R. M., Dahlgren, L., Siegfried, B. D. \& Ellis, M. D. Acaricide, fungicide and drug interactions in honey bees (Apis mellifera). PLoS ONE 8 , e54092 (2013)

45. de Madrid, A. T. \& Porterfield, J. S. A simple micro-culture method for the study of group B arboviruses. Bull. World Health Organ. 40, 113-121 (1969).

46. Zivanov, J. et al. New tools for automated high-resolution cryo-EM structure determination in RELION-3. Elife 7, e42166 (2018).

47. Punjani, A., Rubinstein, J. L., Fleet, D. J. \& Brubaker, M. A. cryoSPARC: algorithms for rapid unsupervised cryo-EM structure determination. Nat. Methods 14, 290-296 (2017).

48. Asarnow, D., Palovcak, E. \& Cheng, Y. UCSF pyem v0.5 (Zenodo, 2019); https://doi.org/10.5281/zenodo. 3576630

49. Pettersen, E. F. et al. UCSF chimera-a visualization system for exploratory research and analysis. J. Comput. Chem. 25, 1605-1612 (2004).

50. Emsley, P. \& Cowtan, K. Coot: model-building tools for molecular graphics. Acta Crystallogr. D Struct. Biol. 60, 2126-2132 (2004)

51. Liebschner, D. et al. Macromolecular structure determination using X-rays, neutrons and electrons: recent developments in Phenix. Acta Crystallogr. D Struct. Biol. 75, 861-877 (2019).

52. Wagner, T. et al. SPHIRE-crYOLO is a fast and accurate fully automated particle picker for cryo-EM. Commun. Biol. 2, 218 (2019).

53. Murshudov, G. N. et al. REFMAC5 for the refinement of macromolecular crystal structures. Acta Crystallogr. D Biol. Crystallogr. 67, 355-367 (2011)

54. Burnley, T., Palmer, C. M. \& Winn, M. Recent developments in the CCP-EM software suite. Acta Crystallogr. D Struct. Biol. 73, 469-477 (2017)

55. Winter, G., Lobley, C. M. \& Prince, S. M. Decision making in xia2. Acta Crystallogr. D Biol. Crystallogr. 69, 1260-1273 (2013).

56. Winter, G. xia2: an expert system for macromolecular crystallography data reduction. J. Appl. Crystallogr. 43, 186-190 (2009).

57. Schmitz, K. R., Bagchi, A., Roovers, R. C., van Bergen en Henegouwen, P. M. \& Ferguson, K. M. Structural evaluation of EGFR inhibition mechanisms for nanobodies/VHH domains. Structure 21, 1214-1224 (2013).

58. Kovalevskiy, O., Nicholls, R. A., Long, F., Carlon, A. \& Murshudov, G. N. Overview of refinement procedures within REFMAC5: utilizing data from different sources. Acta Crystallogr. D Struct. Biol. 74, 215-227 (2018).
59. Joosten, R. P., Long, F., Murshudov, G. N. \& Perrakis, A. The PDB_REDO server for macromolecular structure model optimization. IUCrJ 1, 213-220 (2014).

60. Davis, I. W. et al. MolProbity: all-atom contacts and structure validation for proteins and nucleic acids. Nucleic Acids Res. 35, W375-W383 (2007).

61. Painter, J. \& Merritt, E. A. TLSMD web server for the generation of multi-group TLS models. J. Appl. Crystallogr. 39, 109-111 (2006).

62. McCoy, A. J. et al. Phaser crystallographic software. J. Appl. Crystallogr. 40, 658-674 (2007).

\section{Acknowledgements}

This work was supported by the Rosalind Franklin Institute, funding delivery partner EPSRC. PPUK is funded by the Rosalind Franklin Institute EPSRC grant no. EP/ S025243/1. J.H.N., A.L.B., P.J.H., M.W. and P.W. are supported by Wellcome Trust (100209/Z/12/Z). X-ray data were obtained using Diamond Light Source COVID-19 Rapid Access time on Beamline I03 (proposal MX27031). T.M. is supported by Cancer Research UK grants C20724/A14414 and C20724/A26752 (to C. Siebold). J.G.-J. is funded by the National Secretariat of Education (Senescyt-IFTH), Ecuador. H.M.E.D. and J. Ren are supported by the Wellcome Trust (101122/Z/13/Z) and D.I.S. by the UK Medical Research Council (MR/N00065X/1). D.I.S., P.R. and A.R.T. are funded by the Chinese Academy of Medical Sciences (CAMS) Innovation Fund for Medical Science (CIFMS), China (grant no. 2018-I2M-2-002). T.K.T. is funded by the EPA Cephalosporin Fund and The Townsend-Jeantet Charitable Trust (charity no. 1011770). The core virus neutralization facility is supported by gifts to the Oxford COVID-19 Research Response Fund. M.L.K. is supported by the Biotechnology and Biological Sciences Research Council (BBSRC; BB/M011224/1). EM results were obtained at the cryo-EM facility (OPIC) in the Division of Structural Biology, University of Oxford, part of the UK Centre of Instruct-ERIC, and the national EM facility at Diamond, eBIC, through rapid access proposal BI27051. We thank colleagues at the SGC (Oxford) mass spectrometry service for their assistance.

\section{Author contributions}

J.H. identified the parent nanobody and carried out maturation. R.R.R., H.M.E.D., T.M., M.W., D.K.C., V.K.V., J. Radecke, L.C., P.N.M.S. and D.I.S. performed the EM studies. J.H., A.L.B., H.M., J. Ren, D.Z., L.M., Y.Z., D.I.S. and J.H.N. performed crystallography and biophysics. J.H., A.L.B., T.M., T.K.T., R.R., P.J.H., P.N.W., M.D. and P.J.H. produced proteins for the experiments. T.K.T., P.R. and A.R.T. designed the RBD construct for cell surface expression and carried out the cell-based ACE2 competition assays. J.A.T., K.R.B., N.C., M.J.E., M.W.C., J.G.-J., M.L.K. and W.J. carried out neutralization assays. J.H.N. wrote the manuscript with input from all authors. J.H., R.J.O. and J.H.N. designed the study. All authors analyzed the data.

\section{Competing interests}

The Rosalind Franklin Institute has filed a patent on the H11-H4 and H11-D4 nanobodies. J.H., J.H.N., R.J.O. and D.I.S. are named as inventors. The other authors declare no competing interests.

\section{Additional information}

Extended data is available for this paper at https://doi.org/10.1038/s41594-020-0469-6. Supplementary information is available for this paper at https://doi.org/10.1038/ s41594-020-0469-6.

Correspondence and requests for materials should be addressed to R.J.O. or J.H.N

Peer review information Peer reviewer reports are available. Inês Chen was the primary editor on this article and managed its editorial process and peer review in collaboration with the rest of the editorial team.

Reprints and permissions information is available at www.nature.com/reprints. 
a
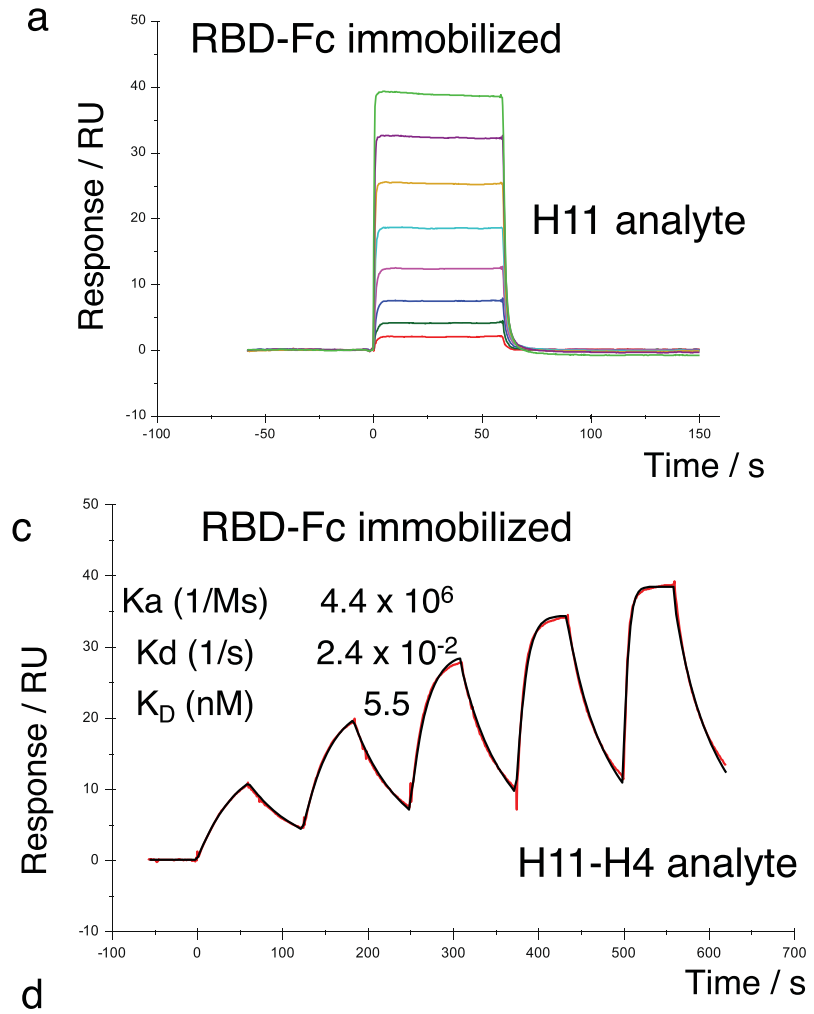

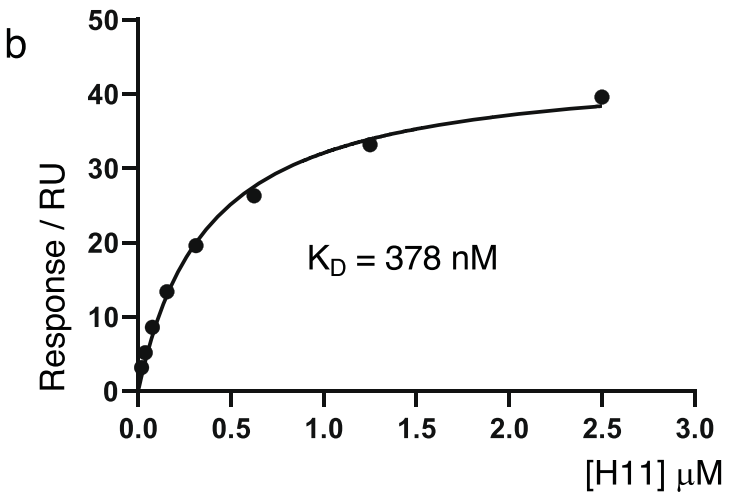

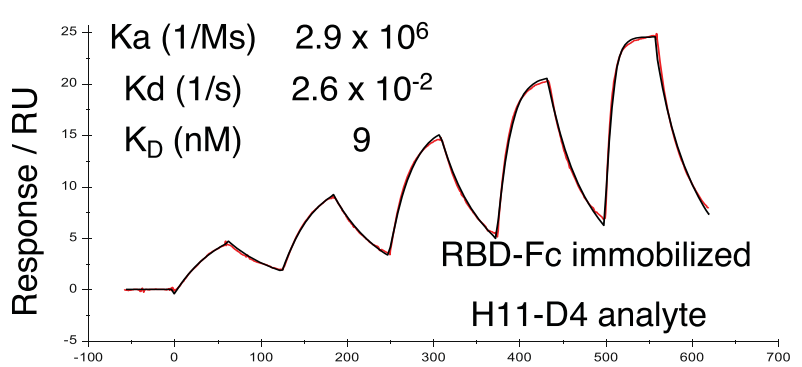

Time / s

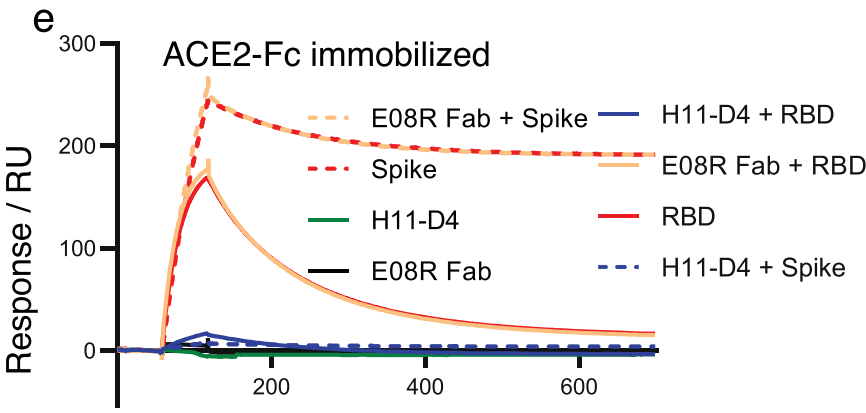

Time / s
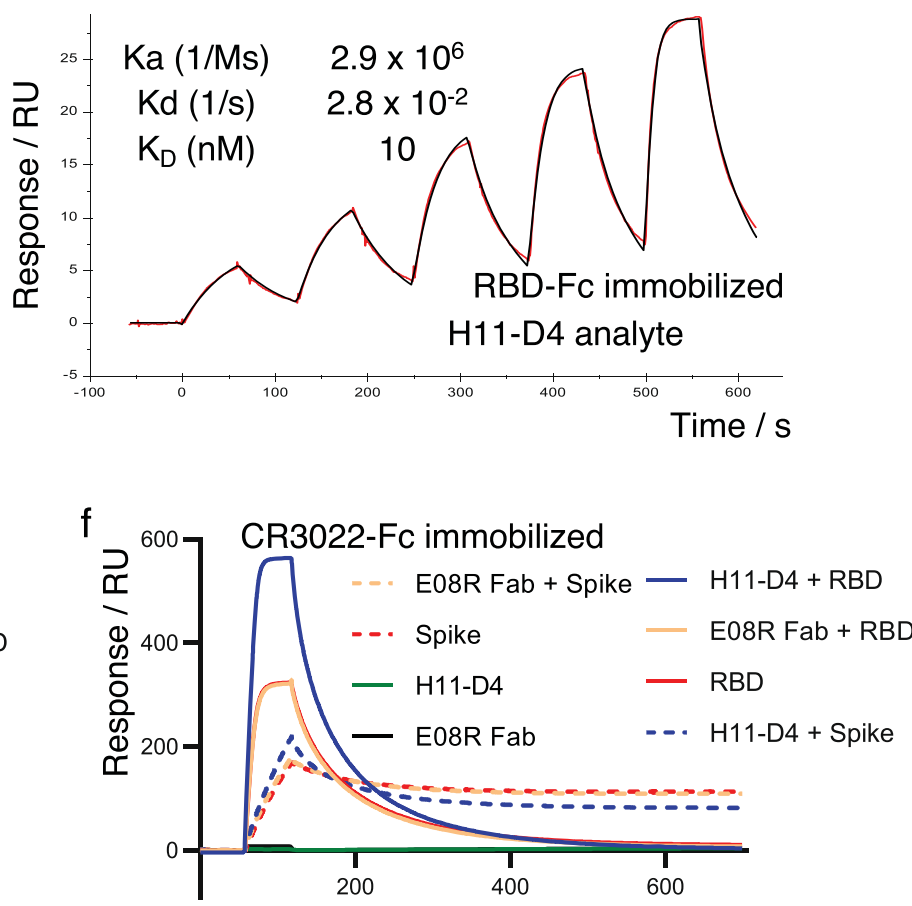

Time / s

Extended Data Fig. 1 | Biophysics of the nanobody binding to RBD. (a) Raw sensorgrams for the $\mathrm{H} 11$ parent nanobody. (b) $\mathrm{K}_{\mathrm{D}}$ of the $\mathrm{H} 11$ parent nanobody. (c) H11-H4 binding to RBD-Fc, repeat of Fig. 2b. (d) H11-H4 binding to RBD-Fc. (e) Binding of analytes to ACE2-Fc immobilized on the chip. H11-D4 behaved identically to H11-H4 (Fig. 2c). (f) Binding of analytes to CR2022-Fc immobilized on the chip. H11-D4 behaved identically to H11-H4 (Fig. 2d). 
a
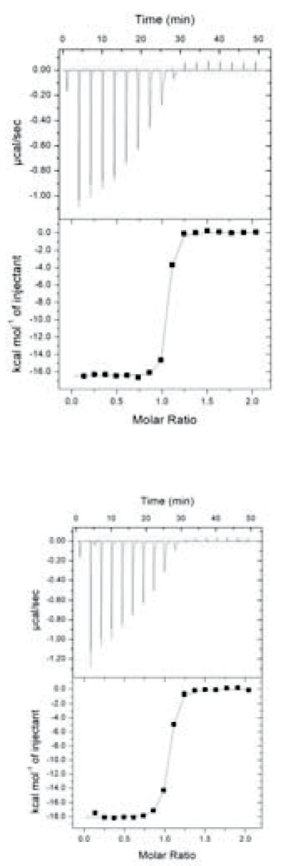

b
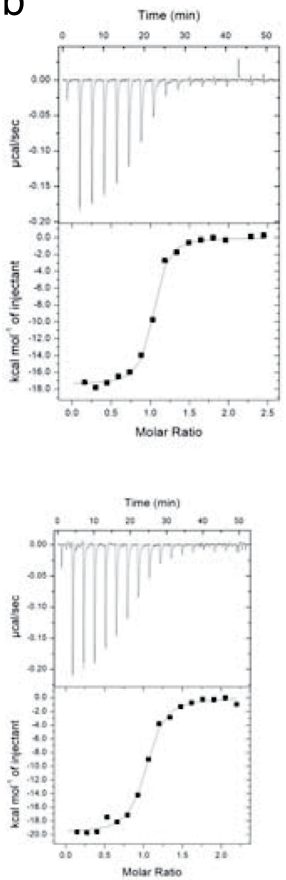
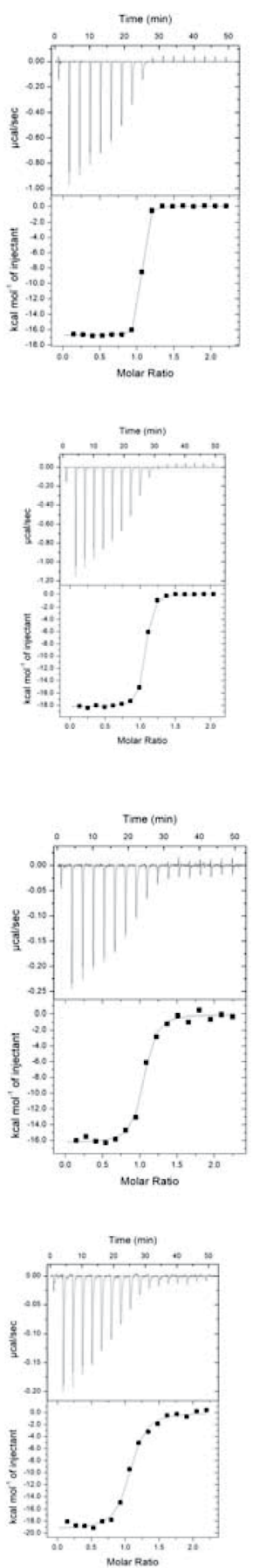

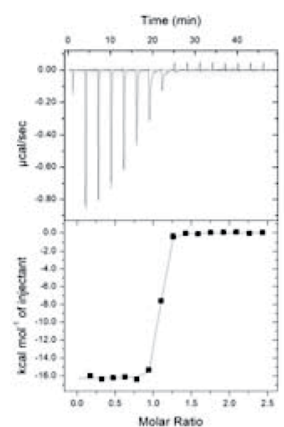

RBD (cell)

H11-H4 (syringe) @ $298 \mathrm{~K}$

$\mathrm{n}=1.01 \pm 0.01$

$\mathrm{K}_{\mathrm{D}}=11.8 \pm 1.5 \mathrm{nM}$

$\Delta \mathrm{H}=-69.0 \pm 0.6 \mathrm{kJmol}^{-1}$

$\Delta \mathrm{S}=-79 \pm 1 \mathrm{Jmol}^{-1} \mathrm{~K}^{-1}$

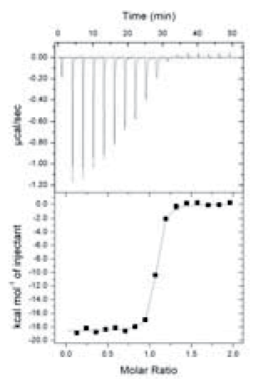

RBD (cell)

H11-D4 (syringe) @ $298 \mathrm{~K}$

$\mathrm{n}=1.01 \pm 0.01$

$\mathrm{K}_{\mathrm{D}}=39.2 \pm 2 \mathrm{nM}$

$\Delta \mathrm{H}=-76.1 \pm 0.6 \mathrm{kJmol}^{-1}$

$\Delta S=-114 \pm 1 \mathrm{Jmol}^{-1} \mathrm{~K}^{-1}$

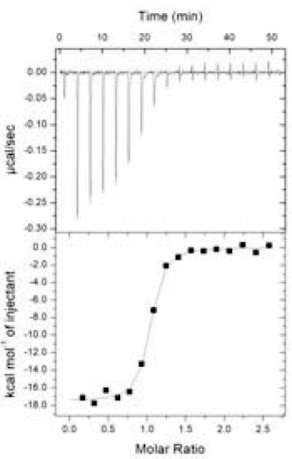

Spike (cell)

H11-H4 (syringe) @ $298 \mathrm{~K}$

$\mathrm{n}=0.984 \pm 0.002$

$\mathrm{K}_{\mathrm{D}}=44 \pm 3 \mathrm{nM}$

$\Delta \mathrm{H}=-74 \pm 1 \mathrm{kJmol}^{-1}$

$\Delta \mathrm{S}=-107 \pm 4 \mathrm{Jmol}^{-1} \mathrm{~K}^{-1}$

Extended Data Fig. 2 | ITC measurements of nanobodies binding to RBD or Spike. (a) Three independent ITC measurement of H11-H4 and H11-D4 binding to RBD. The errors are s.e.m. for three independent experiments, including the one shown in Fig. 2d. (b) As above, but with the Spike protein. 

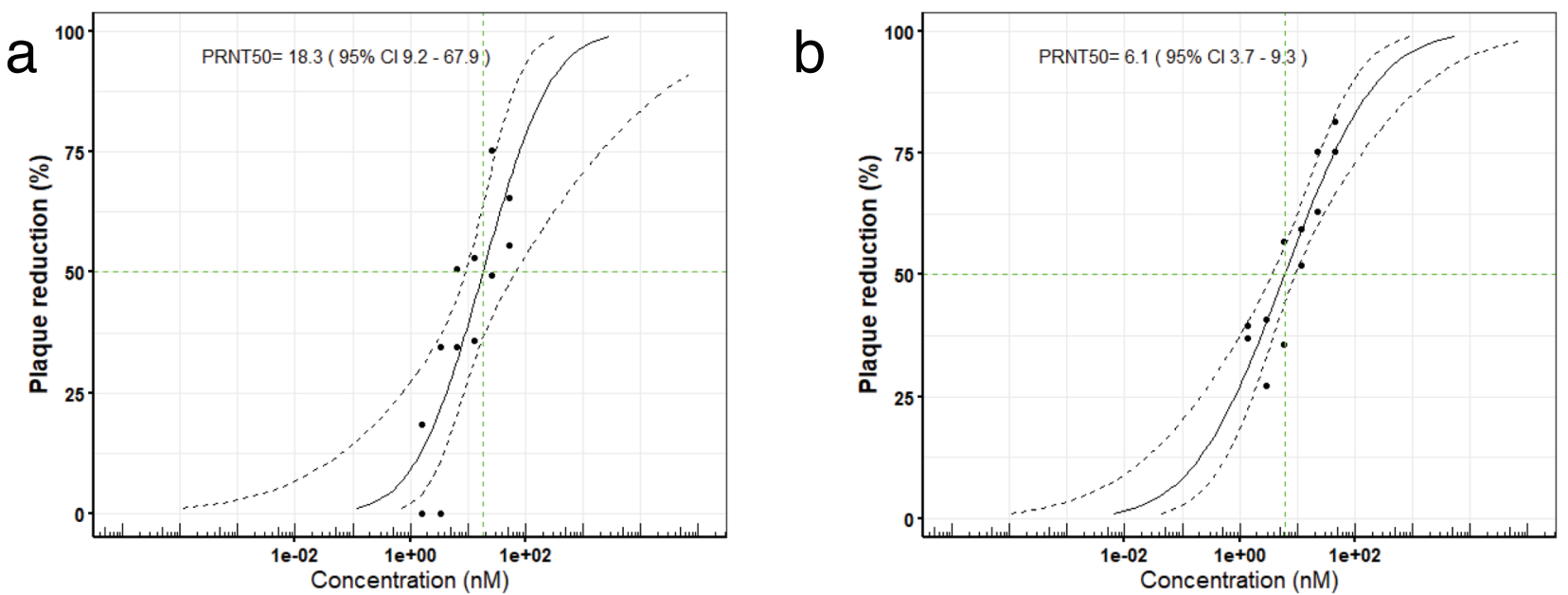

C

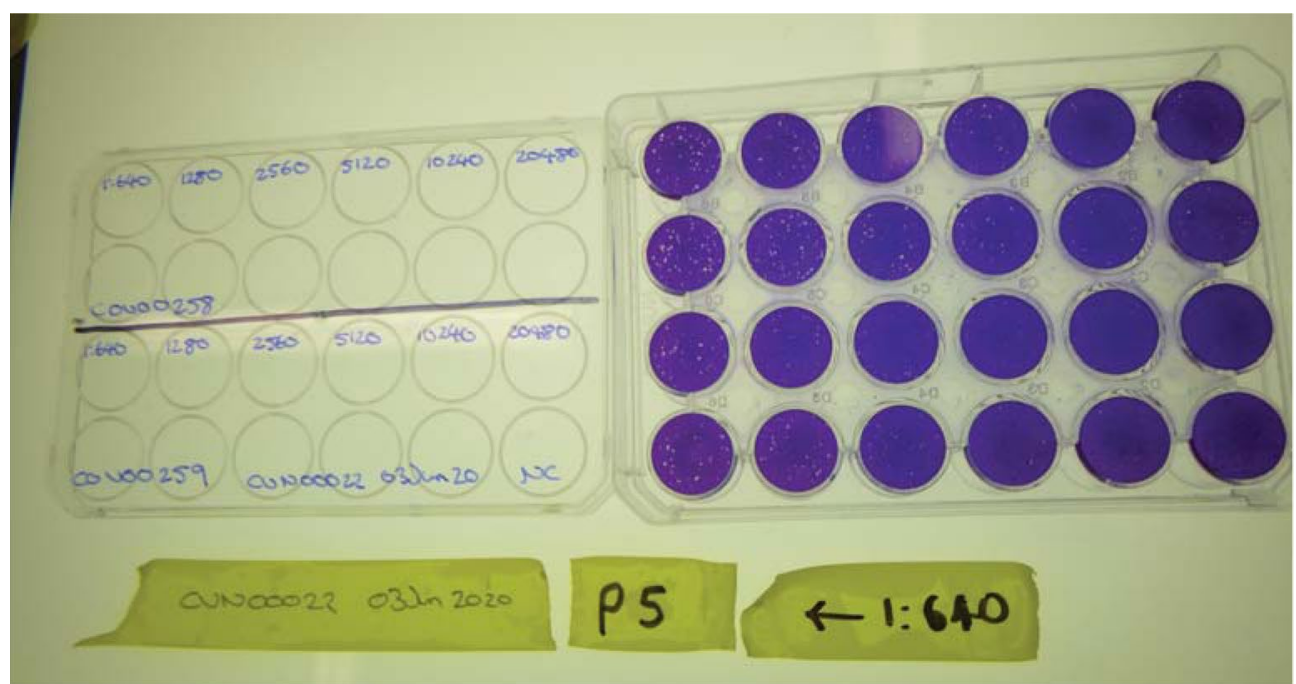

\begin{tabular}{|l|l|l|}
\hline H11-D4-FC fraction B9 e $2.8 \mathrm{mg} / \mathrm{ml}\left(2^{\text {nd }} \mathrm{Batch}\right)$ & COV00258 & CVN00022 \\
\hline $\mathrm{H} 11-\mathrm{H} 4-\mathrm{FC}$ \& $2.46 \mathrm{mg} / \mathrm{ml}\left(2^{\text {nd }}\right.$ Batch $)$ & COV00259 & CVN00022 \\
\hline
\end{tabular}

Extended Data Fig. 3 | Neutralization of live virus at Public Health England. The percentage reduction in plaques arising from virus is plotted againt increasing (left to right) concentration of (a) H11-H4-Fc (6 nM, $95 \% \mathrm{Cl} 3-9 \mathrm{nM})$ (b) H11-D4-Fc (18 nM, $95 \% \mathrm{Cl} 9-68 \mathrm{nM})$. The confidence intervals are shown as dashed lines. In Figure 3d,e 5d \% infectivity (\% infectivity $=100-\%$ plaque reduction) is plotted against decreasing (left to right) concetration of the agent. (c) The experimental plate with the codes noted below. The plaques caused by the virus are visible. 

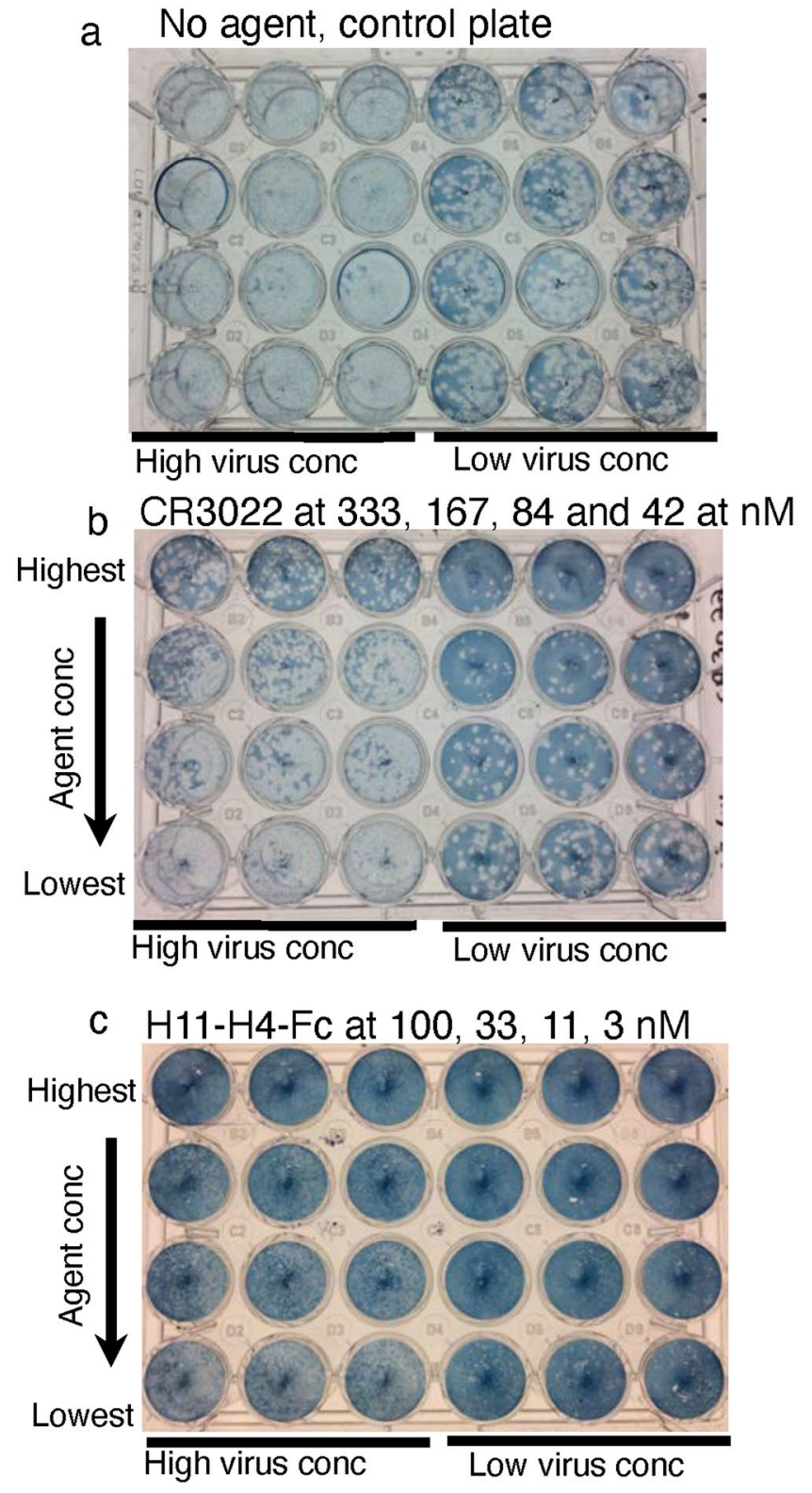
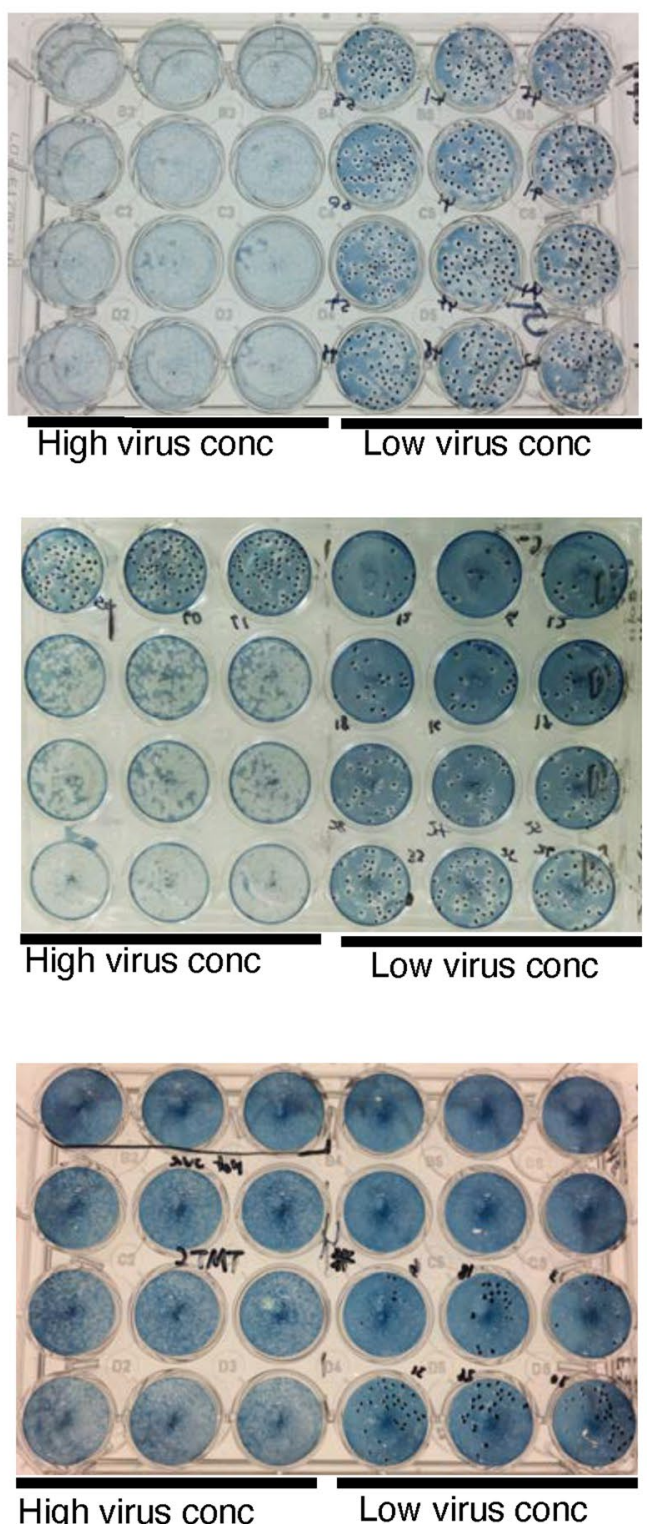

High virus conc

Low virus conc

$33,11,3 \mathrm{nM}$

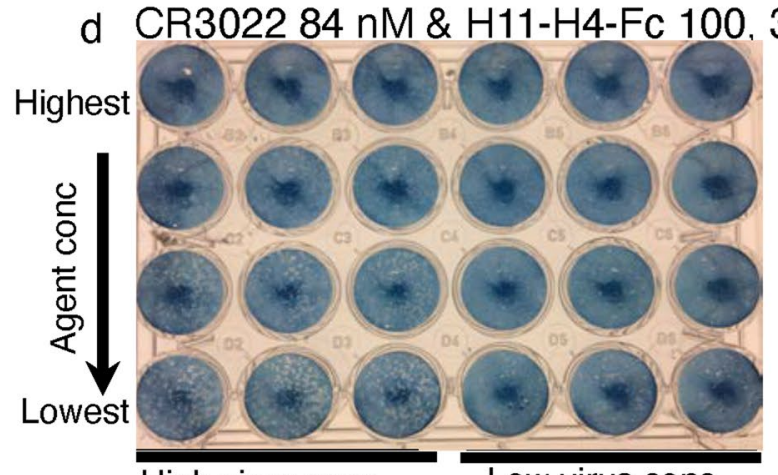

High virus conc

Low virus conc

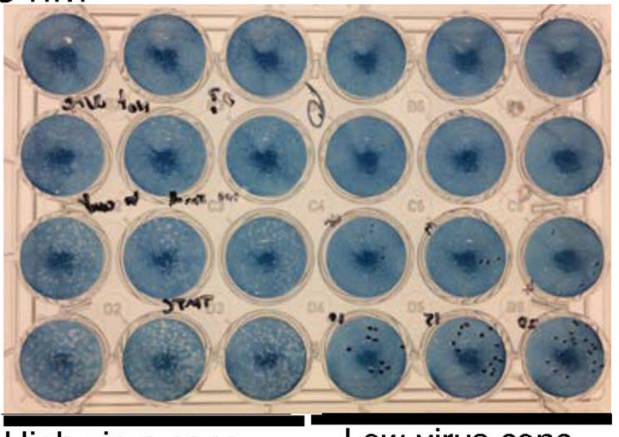

High virus conc

Extended Data Fig. 4 | Neutralization of live virus at Oxford University. The concentration of neutralizing agent was held constant across a row and decreased on subsequent rows. The agent was tested against high and low virus concentrations. (a) Control plate no agent. (b) CR3022. (c) H11-H4-Fc. (d) $\mathrm{H} 11-\mathrm{H} 4-\mathrm{Fc}$ varied and CR3022 held constant at $84 \mathrm{nM}$. Images on left show plaques before pen counting, on the right the pen counts are shown. 
a

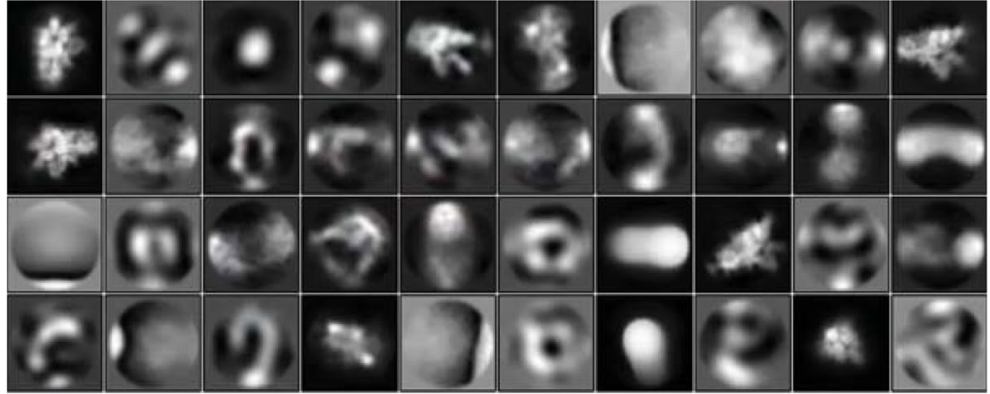

C

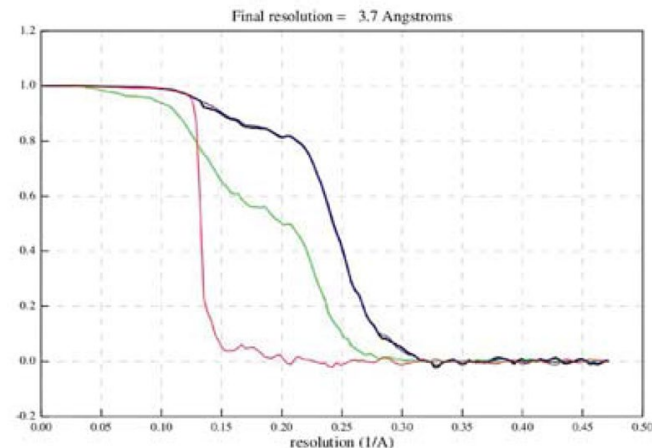

b
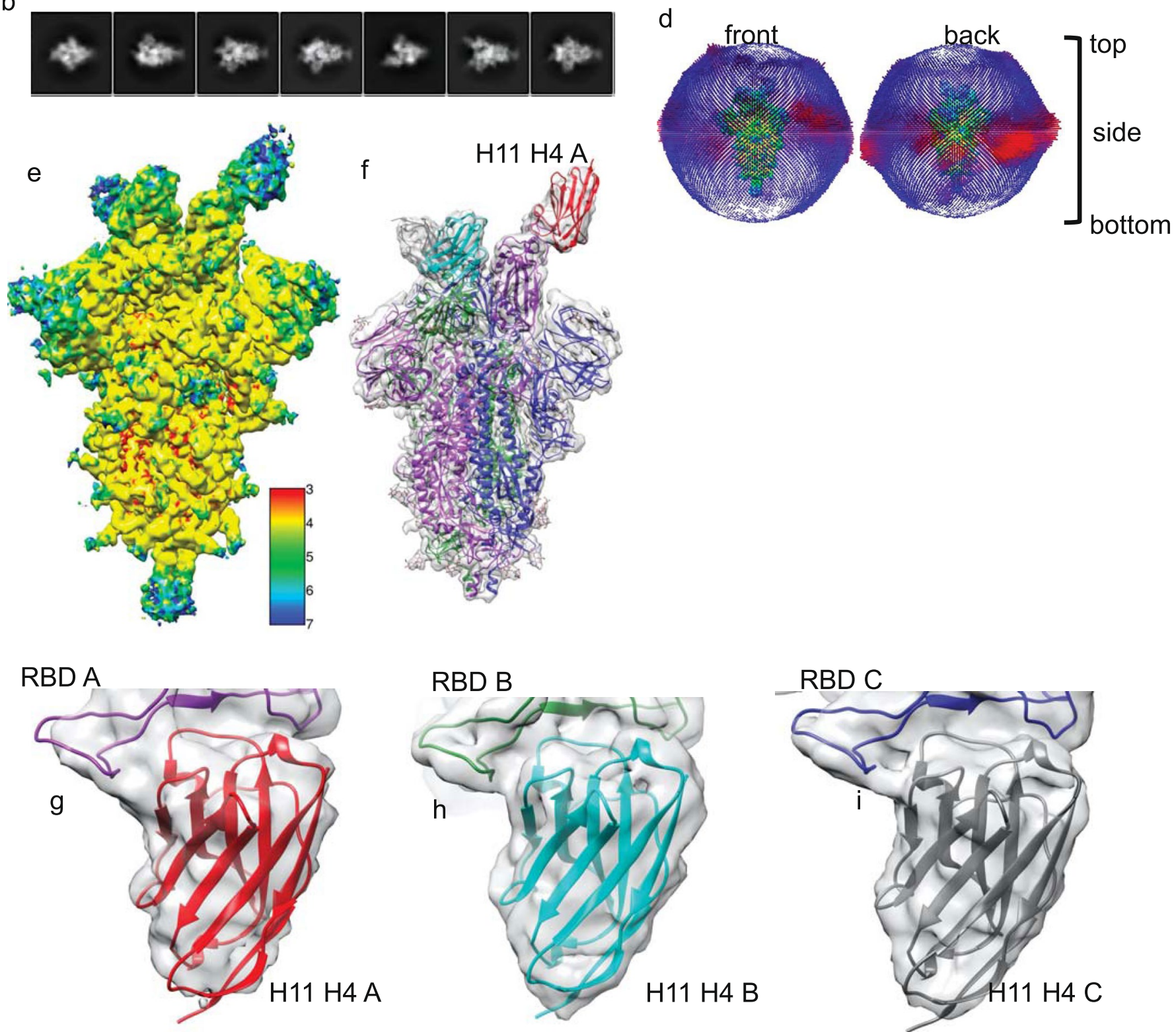

Extended Data Fig. 5 | Cryo-EM of the H11-H4-Spike complex. (a) Unbiased 2D class averages of the complex. (b) 2D class averages selected for further processing. (c) FSC resolution criteria. (d) Particle orientation distribution for the final map showed no preferred orientation. (e) Final map colored according to local resolution. (f) Ribbon diagram of the complex and map (gray) contoured at $4 \sigma$ Chimera $^{49}$. (g-i) The three H11-H4 nanobodies with map contoured at $4 \sigma$ Chimera $^{49}$. Maps in $\mathrm{f}-\mathrm{i}$ used amplitudes scaled based on the refined coordinates using LocScale ${ }^{63}$. 


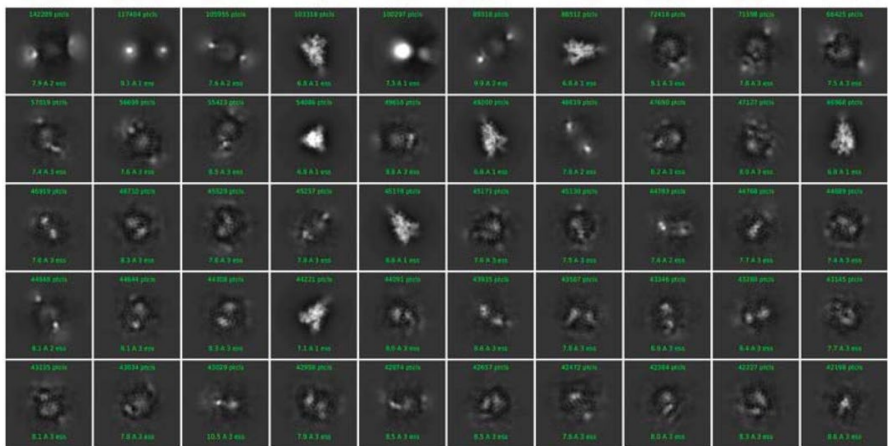

b

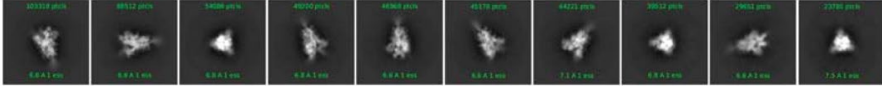

e

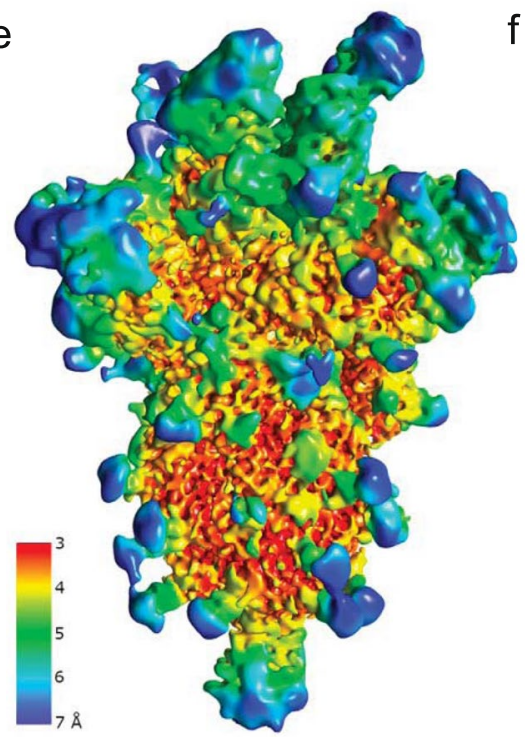

RBD A
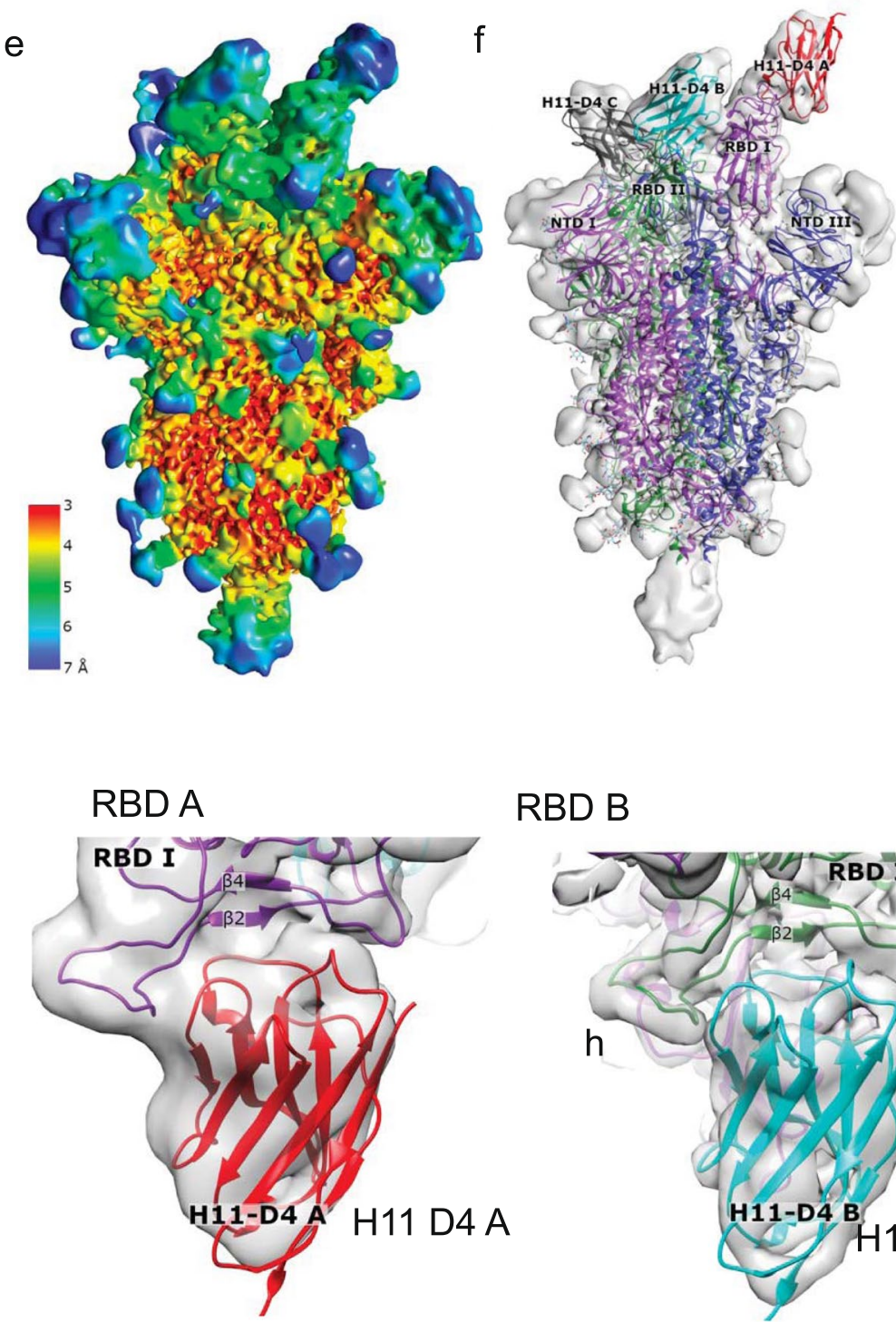

RBD B
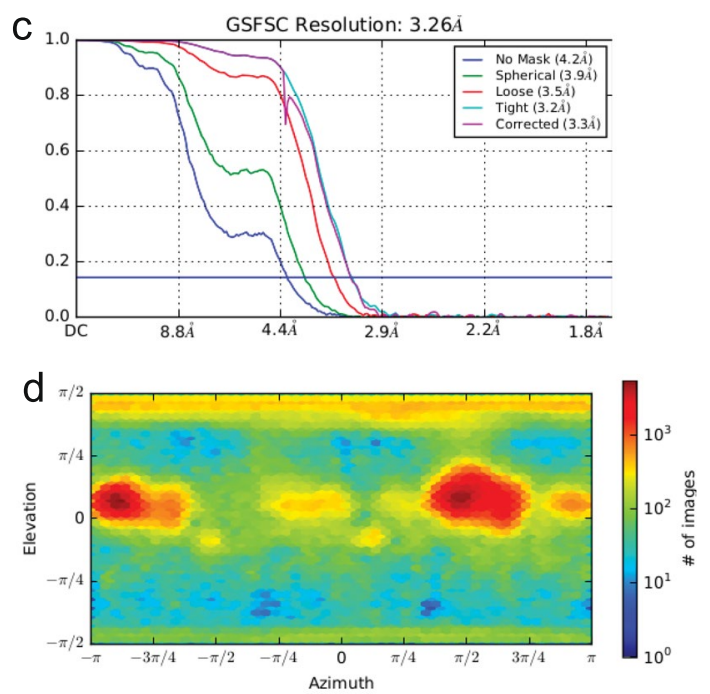

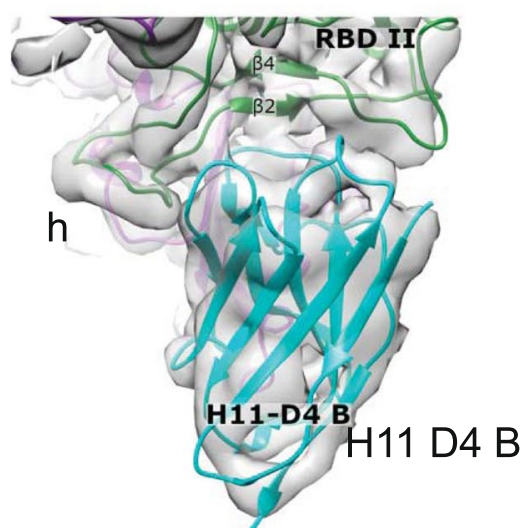

RBD C

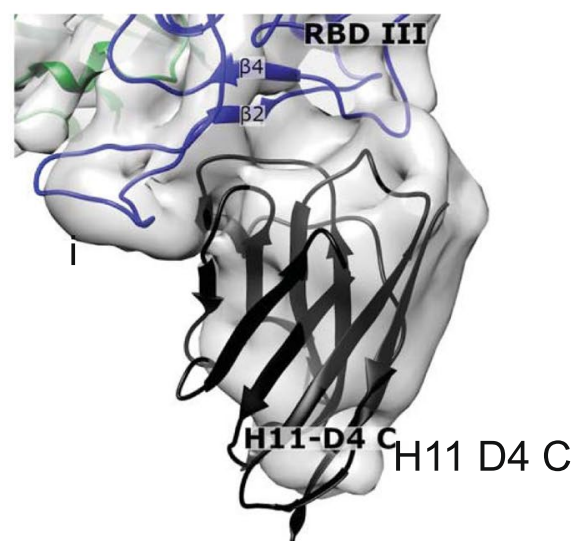

Extended Data Fig. 6 | Cryo-EM of the H11-D4-Spike complex. (a) Unbiased 2D class averages of the complex. (b) 2D class averages selected for further processing. (c) FSC resolution criteria. (d) Particle orientation distribution for the final map showed no preferred orientation. (e) Final map colored according to local resolution. (f) Ribbon diagram of the complex and map (grey) contoured at $3.8 \sigma$ Chimera ${ }^{49}$. (g-i) The three H11-D4 nanobodies with map contoured at $3.8 \sigma$ Chimera $^{49}$. Maps in $\mathrm{f}-\mathrm{i}$ used amplitudes scaled based on the refined coordinates using LocScale ${ }^{63}$. 

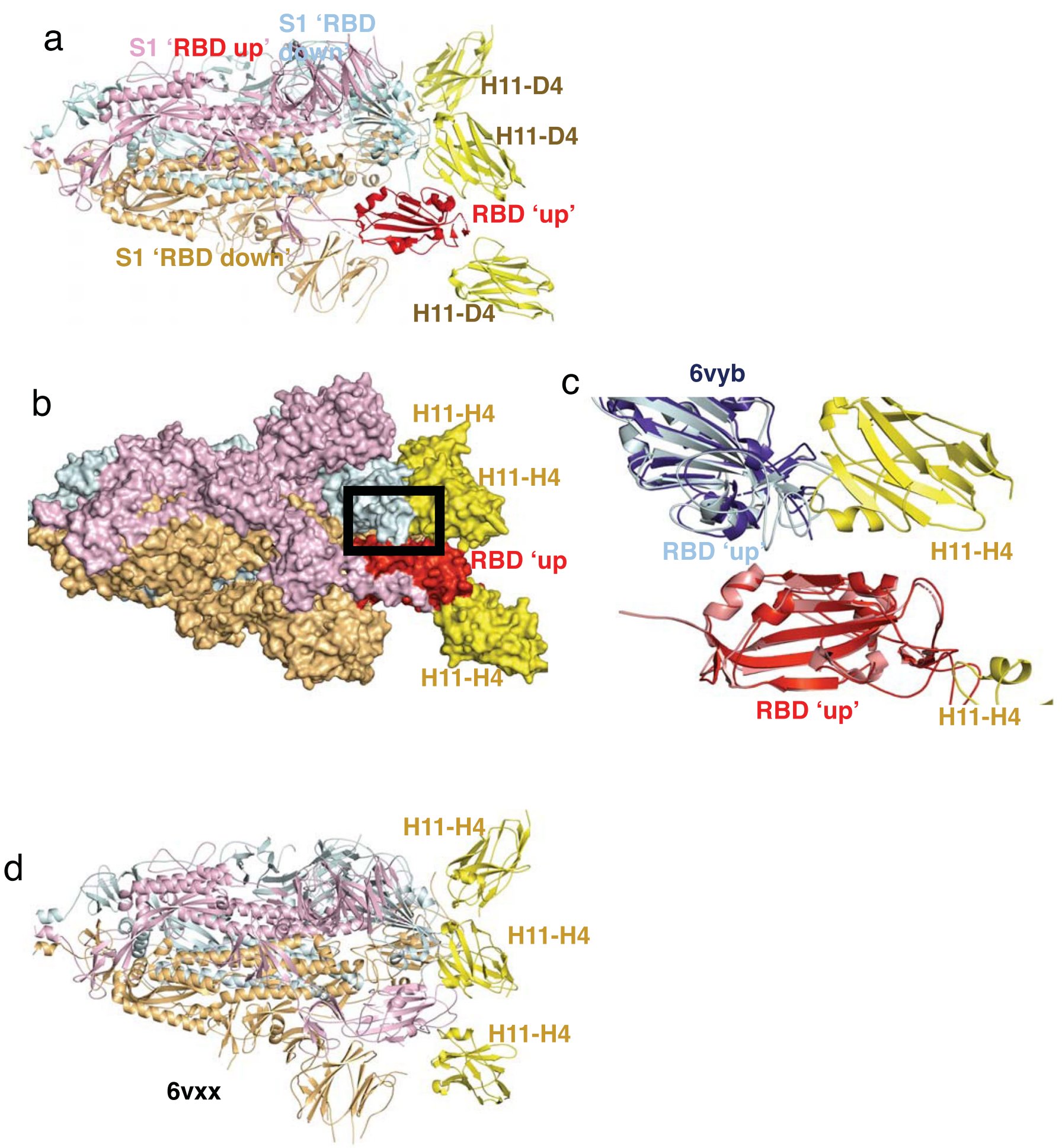

Extended Data Fig. 7 | Further analysis of the cryo-EM nanobody-Spike complexes. (a) The H11-D4-Spike complex is colored as Fig. 4a. (b) The H11-H4Spike shown in surface and colored in Fig. 4a, has revealed there is an interaction between H11-H4 bound to a down subunit and the up RBD. (c) A close of up of the interaction shown in Extended Data Fig. 4a. Shown in dark blue is the down RBD from the Spike structure (PDB 6vyb ${ }^{13}$. The down RBD has shifted by around $2 \AA$. (d) A model constructed from the closed structure of the Spike bound to $\mathrm{H} 11-\mathrm{H} 4$ revealed no clash, indicating the nanobody will recognize this form too. The model was constructed by superimposing the H11-H4-RBD complex onto the EM structure of the closed form of Spike (PDB 6vxx ${ }^{13}$ ). 

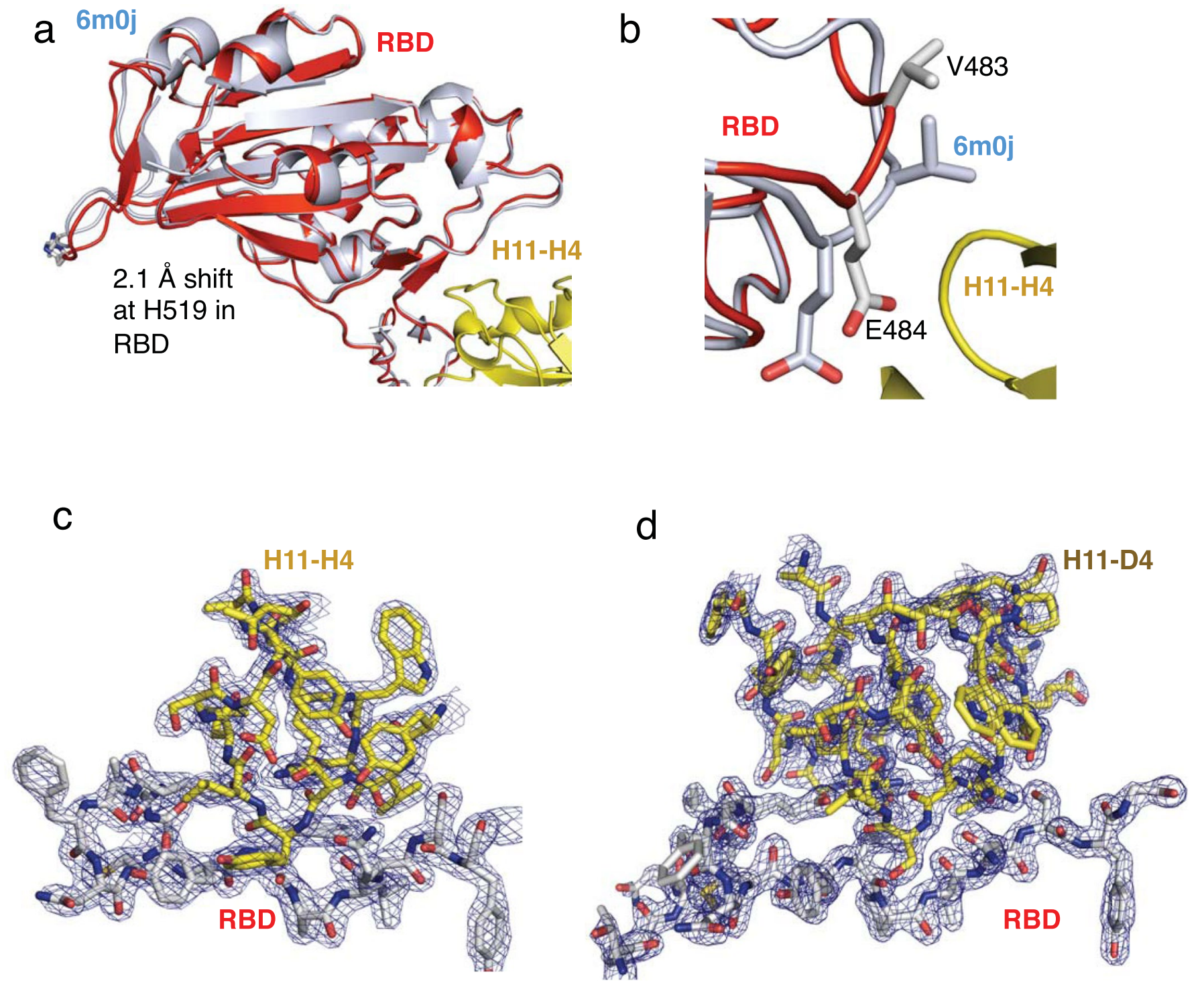

Extended Data Fig. 8 | Further structural analysis of nanobody-RBD crystal structures. ((a) Superimposing the RBDs from the complex with ACE2 ${ }^{29}$ (colored bluewhite PDB $6 \mathrm{mOj}$ ) and the complex with $\mathrm{H} 11-\mathrm{H} 4$, using residues 484 to 510 , indicated a hinging movement occurs within the RBD. As a result of this hinge, the $\mathrm{C} \alpha$ of His 519 has shifted $2.1 \AA$. (b) The binding of $\mathrm{H} 11-\mathrm{H} 4$ results in local shifts at Val483 of the RBD from the ACE2 complex PDB $60 \mathrm{~J}^{29}$ (c) 2Fo-Fc electron density map contoured at $2 \sigma$ for residues at the H11-H4-RBD interface. (d) 2Fo-Fc electron density map contoured at $2 \sigma$ for residues at the H11-D4-RBD interface. 
a

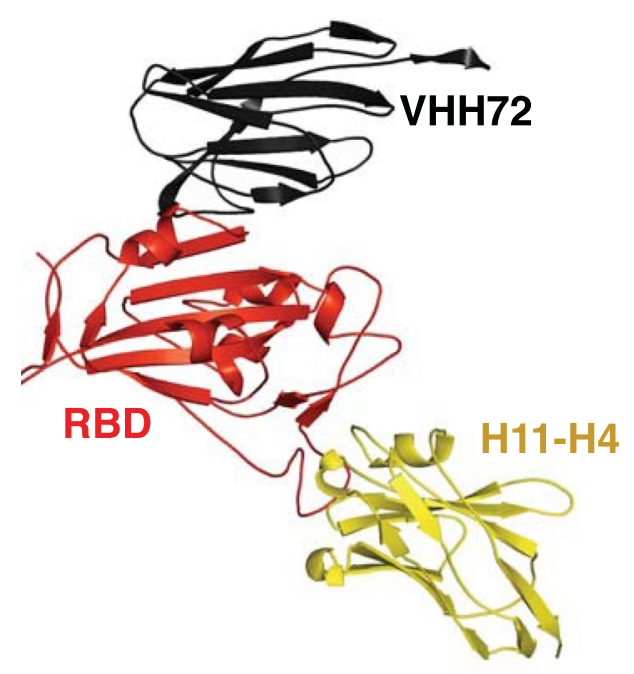

C

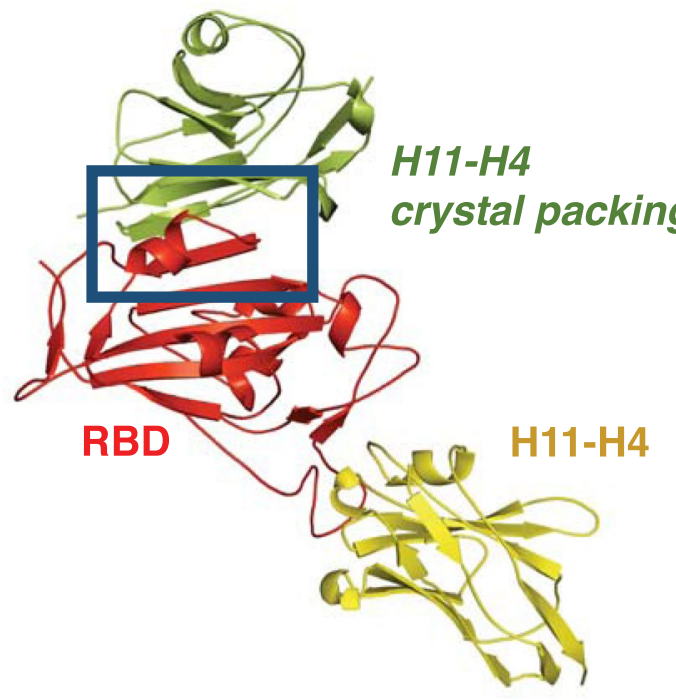

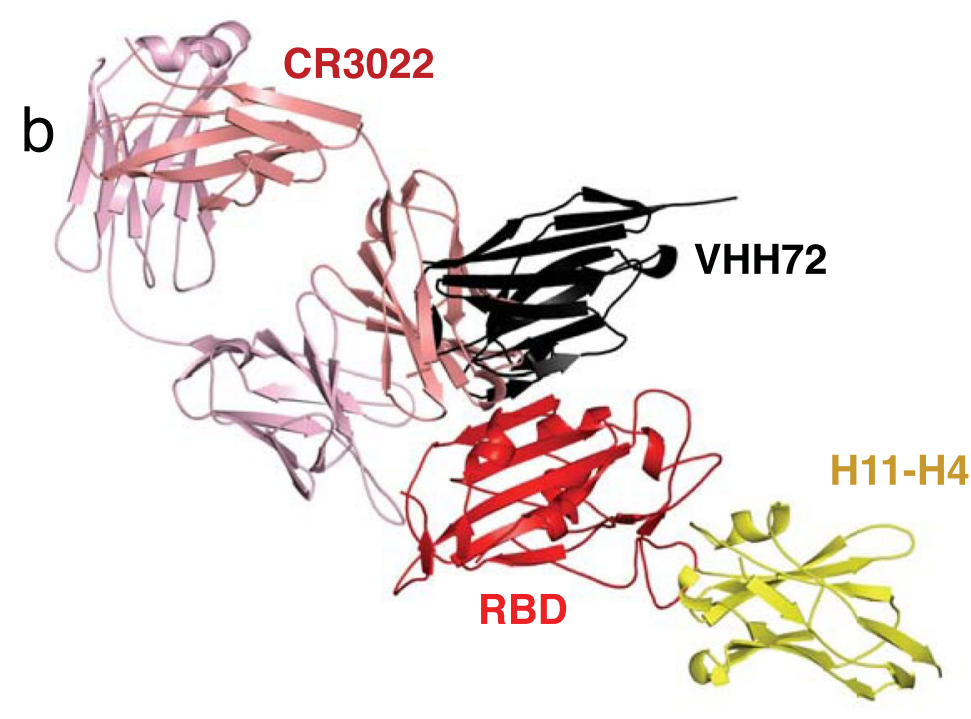

d
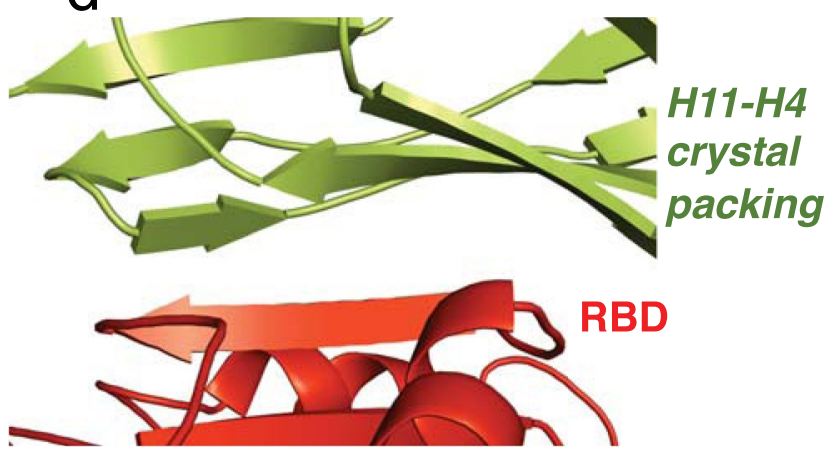

Extended Data Fig. 9 | H11-H4 and VHH72 recognize different epitopes. (a) VHH72 (black) and H11-H4 (yellow) recognize different epitopes on RBD (red). (b) The epitopes for VHH72 and CR3022 (pale pink and lilac) overlap. The figure was generated by superimposing RBD from the CR3002 RBD complex $^{18}$ (PDB 6YLA), VHH72 SARS-CoV-1 RBD complex ${ }^{25}$ (PDB 6WAQ) and the H11-H4-RBD complex. (c) Analysis of the crystal packing in the $\mathrm{H} 11-\mathrm{H} 4-\mathrm{RBD}$ complex revealed that the crystal contact uses the same epitope on the RBD. The crystal contact surface used by $\mathrm{H} 11-\mathrm{H} 4$ is different from that used by $\mathrm{VHH} 72$ (the nanobodies are rotated by $180^{\circ}$ around vertical axis that passes through the center of nanobody). (d) Close up of the crystal contact revealed an antiparallel $\beta$-sheet type interaction. 


\section{Reporting Summary}

Nature Research wishes to improve the reproducibility of the work that we publish. This form provides structure for consistency and transparency in reporting. For further information on Nature Research policies, see our Editorial Policies and the Editorial Policy Checklist.

\section{Statistics}

For all statistical analyses, confirm that the following items are present in the figure legend, table legend, main text, or Methods section.

n/a $\mid$ Confirmed

\ The exact sample size $(n)$ for each experimental group/condition, given as a discrete number and unit of measurement

$\square$ A statement on whether measurements were taken from distinct samples or whether the same sample was measured repeatedly

The statistical test(s) used AND whether they are one- or two-sided

Only common tests should be described solely by name; describe more complex techniques in the Methods section.

\A description of all covariates tested

$\square$ A description of any assumptions or corrections, such as tests of normality and adjustment for multiple comparisons

$\square$ A full description of the statistical parameters including central tendency (e.g. means) or other basic estimates (e.g. regression coefficient)

AND variation (e.g. standard deviation) or associated estimates of uncertainty (e.g. confidence intervals)

For null hypothesis testing, the test statistic (e.g. $F, t, r$ ) with confidence intervals, effect sizes, degrees of freedom and $P$ value noted

Give $P$ values as exact values whenever suitable.

\ For Bayesian analysis, information on the choice of priors and Markov chain Monte Carlo settings

$\square \bigotimes$ For hierarchical and complex designs, identification of the appropriate level for tests and full reporting of outcomes

$\square$ Estimates of effect sizes (e.g. Cohen's $d$, Pearson's $r$ ), indicating how they were calculated

Our web collection on statistics for biologists contains articles on many of the points above.

\section{Software and code}

Policy information about availability of computer code

Data collection Xia2, RELION, CRYOSPARC

Data analysis PRISM v8, CCP4, COOT, RELION, CRYOSPARC

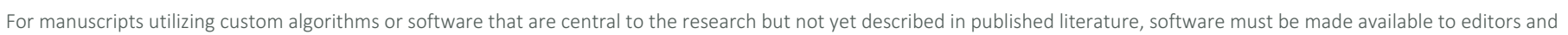

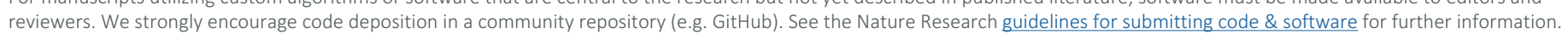

\section{Data}

Policy information about availability of data

All manuscripts must include a data availability statement. This statement should provide the following information, where applicable:

- Accession codes, unique identifiers, or web links for publicly available datasets

- A list of figures that have associated raw data

- A description of any restrictions on data availability

The coordinates and structure factors of the ternary SARS-CoV-2 H11-D4 - RBD - CR3022 complex (6YZ7, 6Z2M) and the binary SARS-CoV-2 H11-D4 - RBD complex (6YZ5). (Figure 4 and 5). EM data has been deposited with code EMD-11068, 6Z43. H11-H4 - RBD complex 6ZH9, H11-H4 - RBD - CR3022 6ZH9, EM H11-H4 - Spike EMD-11218 \& 6ZHD 
Please select the one below that is the best fit for your research. If you are not sure, read the appropriate sections before making your selection.

$\bigotimes$ Life sciences $\quad \square$ Behavioural \& social sciences $\quad \square$ Ecological, evolutionary \& environmental sciences

For a reference copy of the document with all sections, see nature.com/documents/nr-reporting-summary-flat.pdf

\section{Life sciences study design}

All studies must disclose on these points even when the disclosure is negative.

Sample size not applicable
Data exclusions not applicable
Replication not applicable
Randomization not applicable
Blinding not applicable

\section{Reporting for specific materials, systems and methods}

We require information from authors about some types of materials, experimental systems and methods used in many studies. Here, indicate whether each material, system or method listed is relevant to your study. If you are not sure if a list item applies to your research, read the appropriate section before selecting a response.

\begin{tabular}{l|l} 
Materials \& experimental system \\
\hline n/a Involved in the study \\
$\square$ & $\square$ Antibodies \\
$\square$ & $\square$ Eukaryotic cell lines \\
$\square$ & $\square$ Palaeontology and archaeology \\
$\square$ & $\square$ Animals and other organisms \\
$\square$ & $\square$ Clinical data \\
$\square$ & $\square$ Dual use research of concern
\end{tabular}

\begin{tabular}{l|l} 
Methods \\
\hline n/a & Involved in the study \\
$\searrow$ & $\square$ ChIP-seq \\
$\square$ & $\square$ Flow cytometry \\
$\square$ & $\square$ MRI-based neuroimaging
\end{tabular}

Antibodies

Antibodies used

We created the H11-D4 and H11-H4. We report the complex with CR3022.

Validation

This would be the primary citation. CR3022 has been published, Yuan, M. et al. A highly conserved cryptic epitope in the receptorbinding domains of SARS-CoV-2 and SARS-CoV. Science (2020) and Huo et al, https://www.biorxiv.org/ content/10.1101/2020.05.05.079202v1

\section{Eukaryotic cell lines}

Policy information about cell lines

Cell line source(s)

The cells we used for expression of protein and virus neutralization.

Authentication

Commercial and ATCC stocks

Mycoplasma contamination

not applicable

Commonly misidentified lines

(See ICLAC register)

not applicable

Palaeontology and Archaeology

Specimen provenance not applicable 
Tick this box to confirm that the raw and calibrated dates are available in the paper or in Supplementary Information.

Ethics oversight

not applicable

Note that full information on the approval of the study protocol must also be provided in the manuscript.

\section{Animals and other organisms}

Policy information about studies involving animals; ARRIVE guidelines recommended for reporting animal research

\begin{tabular}{|c|c|}
\hline Laboratory animals & None \\
\hline Wild animals & None \\
\hline Field-collected samples & None \\
\hline Ethics oversight & not applicable \\
\hline
\end{tabular}

Note that full information on the approval of the study protocol must also be provided in the manuscript.

\section{Human research participants}

Policy information about studies involving human research participants

Population characteristics

None

Recruitment

None

Ethics oversight

not applicable

Note that full information on the approval of the study protocol must also be provided in the manuscript.

\section{Clinical data}

Policy information about clinical studies

All manuscripts should comply with the ICMJE guidelines for publication of clinical research and a completed CONSORT checklist must be included with all submissions.

Clinical trial registration not applicable

Study protocol not applicable

Data collection not applicable

Outcomes not applicable

\section{Dual use research of concern}

Policy information about dual use research of concern

Hazards

Could the accidental, deliberate or reckless misuse of agents or technologies generated in the work, or the application of information presented in the manuscript, pose a threat to:
No Yes
$\bigotimes \square$ Public health
$\searrow \square$ National security
Х $\square$ Crops and/or livestock
Х $\square$ Ecosystems
$\triangle \square$ Any other significant area 


\section{Experiments of concern}

Does the work involve any of these experiments of concern:
No $\mid$ Yes
$\searrow \square$ Demonstrate how to render a vaccine ineffective
$\searrow \square$ Confer resistance to therapeutically useful antibiotics or antiviral agents
$\bigotimes \square$ Enhance the virulence of a pathogen or render a nonpathogen virulent
Х $\square$ Increase transmissibility of a pathogen
$\searrow \square$ Alter the host range of a pathogen
$\searrow \square$ Enable evasion of diagnostic/detection modalities
Х $\square$ Enable the weaponization of a biological agent or toxin
$\searrow \square$ Any other potentially harmful combination of experiments and agents 